\title{
Workshop - Ökonomische Steuerungsmechanismen: Beispiel Hämatologie und Onkologie
}

\section{Daniel Strech $^{\mathrm{a}} \quad$ Uwe Deh $^{\mathrm{b}}$ Stephan Schmitz ${ }^{\mathrm{c}} \quad$ Irmtraut Gürkan $^{\mathrm{d}} \quad$ Helmut Ostermann ${ }^{\mathrm{e}}$} Stefan W. Krause ${ }^{f}$

${ }^{a}$ Institut für Geschichte, Ethik und Philosophie der Medizin, Medizinische Hochschule Hannover, Hannover, Deutschland;

${ }^{\mathrm{b}}$ AOK-Bundesverband GbR, Berlin, Deutschland;

${ }^{\mathrm{c}}$ Gemeinschaftspraxis für Onkologie und Hämatologie, Onkologie-Köln, Köln, Deutschland;

${ }^{\mathrm{d}}$ Universitätsklinikum Heidelberg, Heidelberg, Deutschland;

${ }^{e}$ Abteilung Hämatologie/Onkologie, Medizinischen Klinik und Poliklinik III, Klinimum Großhadern,

Ludwig-Maximilians-Universität München, München, Deutschland;

${ }^{f}$ Medizinische Klinik 5, Universitätsklinikum Heidelberg, Heidelberg, Deutschland

\section{Das deutsche System und seine Werturteile im internationalen Vergleich}

\section{Referent: Daniel Strech, Hannover}

Bei Entscheidungen im Gesundheitssystem spielen verschiedene, allgemein akzeptierte und tradierte Wertvorstellungen wie z.B. der Erhalt der Menschenwürde eine Rolle. Spezifischer geht es dabei unter anderem um Werte wie das Wohltuns- und das Nichtschadensgebot, die Respektierung der Patientenautonomie und eine gerechte Verteilung knapper Ressourcen. Darüber hinaus sind diese Werturteile von besonderer ökonomischer Relevanz, insbesondere in den Themenbereichen Priorisierung, Nutzenbewertung und NutzenSchaden-Abwägung.

Ich möchte zwei Typen von praxisrelevanten Werturteilen unterscheiden. Zunächst einmal müssen die für die jeweilige Fragestellung relevanten Werte operationalisiert werden, um so zu relevanten und praxistauglichen Outcome-Parametern bzw. Priorisierungskriterien zu gelangen (Werturteile Typ 1). Weiterhin müssen diese Werte in Konfliktsituationen gegeneinander abgewogen werden (Werturteile Typ 2). Schließlich sollte das Entscheidungsprozedere auch formalen Werten wie Transparenz, Begründung und Partizipation genügen und für Außenstehende nachvollziehbar sein (Abb.1).

\section{Werturteile in der Priorisierung}

Die Zentrale Ethikkommission bei der Bundesärztekammer hat als bislang einzige Institution im Jahr 2007 zu Fragen der Priorisierung medizinischer Leistungen mit der konkreten Benennung von Priorisierungskriterien Stellung genommen
[1]. Mit der Fortentwicklung der in diesem Papier aufgeführten, für eine Priorisierung maßgeblichen Werte (medizinische Bedürftigkeit, zu erwartender medizinischer Nutzen sowie Kosteneffektivität) und den dafür nötigen formalen Grundlagen (etwa Evidenz und Transparenz) befasst sich die Arbeitsgruppe «Priorisierung im Gesundheitswesen» der Bundesärztekammer.

Auch in anderen Ländern werden Fragen der Priorisierung im Bereich der medizinischen Versorgung bereits seit den 1980er Jahren intensiv diskutiert [2-4]. Die Werturteile zu relevanten Priorisierungskriterien weichen im internationalen Vergleich nicht wesentlich von den hierzulande formulierten Kriterien [1] ab, jedoch werden die Priorisierungsbestrebun-

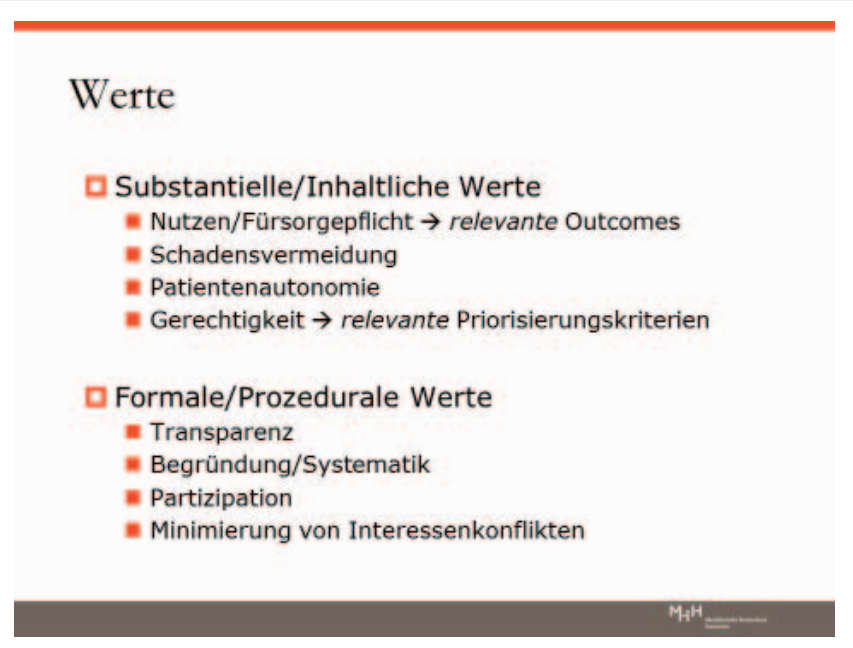

Abb. 1. Werte als Grundlage für Priorisierungen.

\begin{tabular}{ll}
\hline KARGER & $\begin{array}{l}\text { ( ) 2015 S. Karger GmbH, Freiburg } \\
\text { 2296-5270/15/3813-0003\$39.50/0 }\end{array}$ \\
$\begin{array}{l}\text { Fax +497614520714 } \\
\text { Information@Karger.com } \\
\text { www.karger.com }\end{array}$ & $\begin{array}{l}\text { Accessible online at: } \\
\text { www.karger.com/ort }\end{array}$
\end{tabular}




\section{Zwischenfazit: Priorisierung}

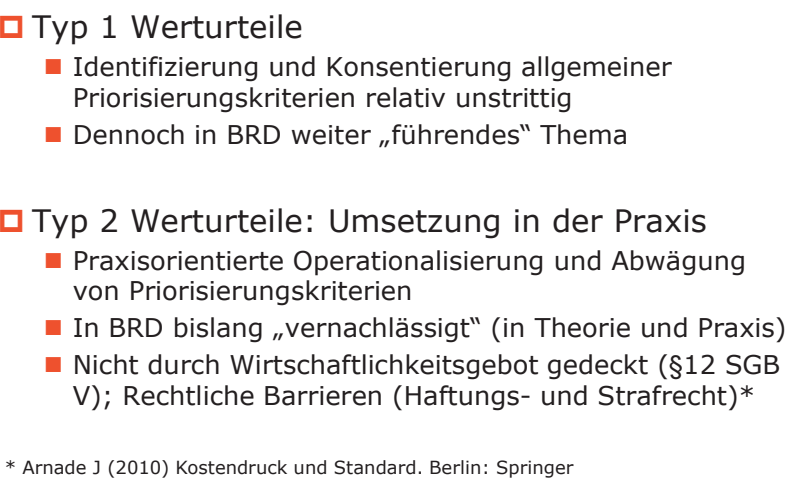

Abb. 2. Entwicklungen in der Priorisierung.

gen recht unterschiedlich umgesetzt. So wurde im US-Staat Oregon eine für die Kostenerstattung von Medicaid verbindliche Priorisierungsliste entwickelt, die sich ausschließlich an der Kosteneffizienz verschiedener medizinischer Maßnahmen orientierte. Diese Liste führte zum Teil zu kontraintuitiven Priorisierungen, musste mehrmals überarbeitet werden und erwies sich schlussendlich als nicht effektiv für eine gesellschaftlich akzeptierte Kostenreduktion. Auch in Großbritannien gibt es bereits seit langem Priorisierungs- und Rationierungsentscheidungen, bei denen allerdings die Kosteneffektivität nicht das ausschließliche Ranking-Kriterium ist. Ein hierzulande häufig diskutiertes Modell kommt aus Schweden. Hier gibt es Priorisierungslisten für bestimmte Fachbereiche, die zunächst als unverbindliche Handlungsempfehlungen zu verstehen sind.

Die einer Priorisierung zugrundeliegenden Typ-1-Werturteile sind in Deutschland weitgehend ausformuliert (Abb. 2). Eine Anwendung der Priorisierungskriterien auf bestimmte medizinische Fragestellungen in Kombination mit Abwägungen zwischen verschiedenen Bedürftigkeiten von Patienten und Nutzenausmaße der jeweiligen Interventionen wird derzeit in Deutschland nicht vorgenommen. Grund dafür dürften auch die bestehenden rechtlichen Barrieren sein [5].

\section{Werturteile in der Nutzenbewertung}

Auch in der Nutzenbewertung medizinischer Maßnahmen spielen Werturteile eine Rolle. Bereits im Vorfeld der NutzenSchaden-Abwägung, etwa bei der Zusammenstellung der dafür nötigen Studiendaten, geht es nicht wertfrei zu. Aufbau, Umfang und Dauer einer kontrollierten Studie werden nicht zuletzt mit Blick auf die Kosten geplant. Zwar sollen möglichst viele Informationen in die Nutzenbewertung einfließen, doch werden andererseits mit guten Gründen hohe Ansprüche an die Qualität der Informationen gestellt (Abb. 3). Insofern geht es nicht ohne eine gewisse Risikobereitschaft bei der Frage, ob und ab welcher Qualität Studieninformationen als

\section{Werturteile im Studieneinschluss}

- Einschlusskriterium: Studienqualität, -validität

$\square$ Wertekonflikt:

- Glaubwürdigkeit von

Informationen

- Information per se

口 Stellschraube:

- Risikobereitschaft

$\square$ Beispiel Mammographiescreening ${ }^{1,2}$

1. Gotzsche PC, Olsen $\mathrm{O}$. Is screening for breast cancer with mammography justifiable? Lancet 2000;355(9198):129-34.

Humphrey, L. L. et al. (2002). "Breast cancer screening: a summary of the evidence for the U.S. Preventive Services Task Force." Ann Intern Med 137: 347-60.

Abb. 3. Werturteile und -konflikte im Umfeld von Studien.

\section{NICE und Werturteile}

\section{$\square$ Social value judgements $(2008)^{1}$}

- Fokus auf Werturteile in Evidenzbewertung (Appraisal)

\section{$\square$ Appraising life-extending, end of life treatments $(2009)^{2}$ \\ - Kurzer Lebenszeitgewinn vor dem Tod hat große subjektive Bedeutung für den einzelnen Patienten \\ - Mögliche ethische Rechtfertigung für Kostenübernahme bei relativ geringer Kosteneffektivität}

1. NICE. Social value judgements. Principles for the development of NICE guidance. Second edition. London: NICE, 2008

. NICE, Appraising life-extending, end of life treatments. A supplementary advice to the Appraisal Committees. London: NICE, 2009

Abb. 4. In die Entscheidungen des NICE fließen auch Werturteile ein.

glaubwürdig anzusehen sind. Ein Beispiel für diesen Wertekonflikt ist das Mammographie-Screening, dessen Nutzen auf Basis identischer Studiendaten von verschiedenen Experten durchaus unterschiedlich bewertet werden kann [6,7]. Offenbar gibt es auch bei der Qualitätsbewertung von Evidenz einen Interpretationsspielraum [8,9]. Die Extraktion und Gewichtung von Daten wird unvermeidbar durch subjektive Werturteile beeinflusst. Ein möglichst systematischer und expliziter Umgang mit diesen Werturteilen kann weiterhin als blinder Fleck in der Methodenforschung zur «evidence-based medicine» gelten [10-12].

Das britische National Institute for Health and Care Excellence (NICE) benennt verschiedene seiner Werturteile explizit (Abb. 4). Der Fokus liegt dabei allerdings auf Werturteilen in der abschließenden Nutzenbewertung nach Erstellung der Studiendossiers.

Das Institut für Qualität und Wirtschaftlichkeit im Gesundheitswesen (IQWiG) hat kein dem NICE-Report entsprechendes Dokument vorgelegt, muss sich aber neuerdings im 
Rahmen der Zusatznutzenbewertung zwangsläufig mit der Frage nach Werturteilen befassen. Das Arzneimittelmarktneuordnungsgesetz (AMNOG) verlangt ausdrücklich eine Abwägung des Zusatznutzens, wobei zwischen erheblichem, beträchtlichem und geringem Zusatznutzen zu differenzieren ist. «Hier fließen Werturteile ein und eine allgemein anerkannte wissenschaftliche Methodik, wie man solche Abwägungen treffen kann, gibt es bislang nicht», hieß es dazu von Seiten des IQWiG im Zusammenhang mit der Nutzenbewertung des Wirkstoffes Ticagrelor [13]. Man habe erläutert, wie man zur Bewertung einzelner Endpunkte und zur Gesamtaussage gelangt sei, und wolle damit einen Anstoß für eine wissenschaftliche Debatte geben. Aus ethischer Sicht erscheint in diesem Zusammenhang mehr Transparenz bei der initialen Entwicklung von Werturteilbegründungen wünschenswert. Im internationalen Vergleich übernimmt das IQWiG hier eine Vorreiterrolle, denn mit Operationalisierungen zu qualitativ hierarchisierenden Nutzenbewertungen gibt es bislang kaum Erfahrungen.

\section{Werturteile zur Über-/Unterversorgung}

In Fragen der Über- und Unterversorgung gibt es bislang keine deutsche Institution, die dazu unter Einbeziehung von Werturteilen Stellung nimmt. Auch in Leitlinien finden sich keine konkreten Hinweise, welche Maßnahmen unter dem Aspekt der Über- oder Unterversorgung zu vermeiden bzw. zu empfehlen sind. Erste Ansätze finden sich allenfalls außerhalb des GKV-Erstattungsbereichs im sogenannten IGeL-
Monitor und auf internationaler Ebene im Rahmen der «Choosing Wisely»-Aktion.

Tendenzen der Überversorgung im Bereich der Medizin sind ein vielfach beschriebenes Problem und finden sich beispielsweise im Bereich von Screening-Untersuchungen [14]. Die daraus resultierende Schlussfolgerung «weniger ist mehr» gründet auf dem Nichtschadensgebot («primum nil nocere») und bräuchte insofern keine weitere (ökonomische) Begründung. Allerdings lassen sich Nutzen und Schaden einer medizinischen Maßnahme eben nur mithilfe von Werturteilen abwägen, weil der Nutzen (etwa in Form eines vermiedenen Todes bei einem von 1000 Teilnehmenden einer fiktiven Früherkennungsmaßnahme) mit dem potentiellen Schaden (z.B. in Form

\section{Der informierte Patient}

These: Man sollte die Abwägung von Nutzen und Schaden immer dem angemessen informierten Patienten überlassen

- Medizinethik: Respekt der Patientenautonomie

$\square$ Mögliche Einwände

- Begrenzte Kompetenz der Patienten

- Begrenzte Kompetenz der aufklärenden Ärzte

Abb. 5. Nutzen-Schaden-Abwägung durch den Patienten.
Abb. 6. NutzenSchaden-Abwägung im Bereich von IGeL-Leistungen.

\section{Abwägung von Nutzen und Schaden einzelner IGeL (nach 5 Bewertungstypen) \\ - In diese Bewertungen fließen "subjektive Wertmaßstäbe" ein}

\begin{tabular}{|l|l|l|l|l|}
\hline & $\begin{array}{l}\text { Keine Hinweise } \\
\text { auf Nutzen }\end{array}$ & $\begin{array}{l}\text { Hinweise auf } \\
\text { geringen Nutzen }\end{array}$ & $\begin{array}{l}\text { Belege für } \\
\text { geringen Nutzen / } \\
\text { Hinweise auf } \\
\text { erheblichen Nutzen }\end{array}$ & \multicolumn{1}{|c|}{$\begin{array}{l}\text { Belege für } \\
\text { erheblichen Nutzen }\end{array}$} \\
\hline $\begin{array}{l}\text { Keine Hinweise } \\
\text { auf Schaden }\end{array}$ & unklar & tendenziell positiv & positiv \\
\hline $\begin{array}{l}\text { Hinweise auf } \\
\text { geringen Schaden }\end{array}$ & tendenziell negativ & unklar & tendenziell positiv \\
\hline $\begin{array}{l}\text { Belege für } \\
\text { geringen Schaden } \\
\text { Hinweise auf } \\
\text { erheblichen Schaden }\end{array}$ & negativ & tendenziell negativ & unklar & positiv \\
\hline $\begin{array}{l}\text { Belege für } \\
\text { erheblichen Schaden }\end{array}$ & negativ & negativ & tendenziell negativ & \\
\hline
\end{tabular}




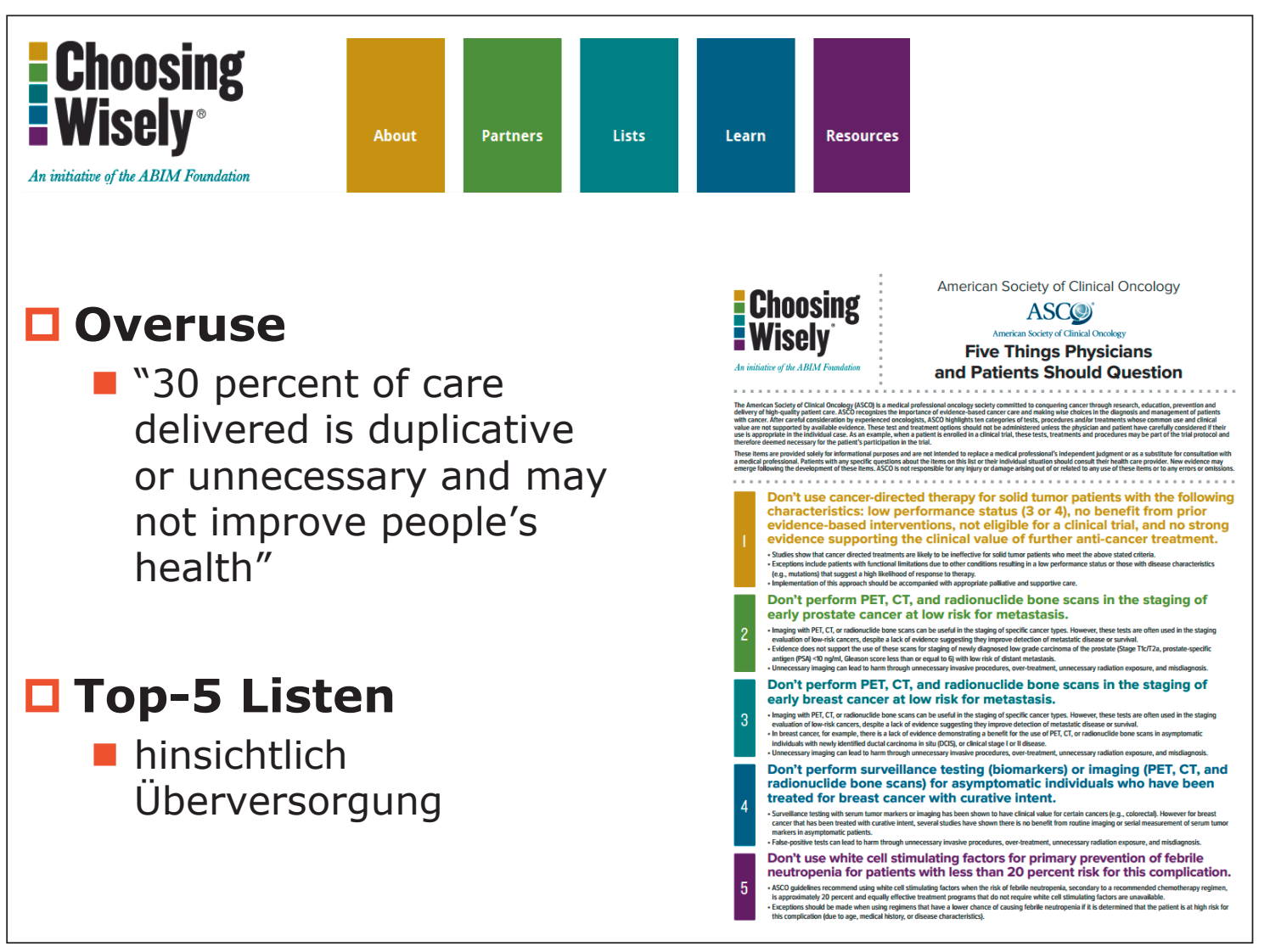

sierungsbestrebungen in den USA.

überflüssiger Anschlussuntersuchungen oder präventiver Therapie mit Nebenwirkungen bei 100 von 1000 Teilnehmenden der gleichen fiktiven Früherkennungsmaßnahme) nicht direkt vergleichbar ist. Aus diesem Grund wird die Nutzen-SchadenAbwägung gern dem «informierten» Patienten überlassen (Abb. 5), was eine hohe Kompetenz sowohl seitens des Patienten als auch seitens des informierenden Arztes voraussetzt.

Etwas anders ist der Umgang mit diesen Werturteilen in Nutzen-Schaden-Abwägungen im Kontext klinischer Forschung. Hier muss zwar ebenfalls für eine gute Information des in Frage kommenden Studienteilnehmers zu Nutzen- und Schadenspotentialen der geplanten Studien gesorgt werden. Allerdings müssen hier im Vorfeld auch die zuständigen Ethikkommissionen, stellvertretend für die potentiellen Studienteilnehmer, beurteilen, ob die Nutzen-Schaden-Bilanz positiv ausfällt und das geplante Vorhaben überhaupt bestimmten Personen zuzumuten ist. Ähnliche Szenarien finden sich bei der Marktzulassung von medizinischen Maßnahmen und bei Fragen der Kostenerstattung durch den Gemeinsamen Bundesausschuss (G-BA).

Es fehlt jedoch ein Gremium, das Werturteile $\mathrm{zu}$ «weniger ist mehr» im Kontext der Regelversorgung vornimmt. Ein erster Schritt in diese Richtung ist der IGeL-Monitor des Medizinischen Dienstes des Spitzenverbandes Bund der Krankenkassen e.V. (MDS), in deren Nutzen-Schaden-Bewertung einzelner IGeL-Leistungen nach eigenen Angaben «subjektive Wertmaßstäbe» einfließen (Abb. 6).
Fazit

$\square$ Ethische und praktische Herausforderungen:

Operationalisierung und Abwägung

- Priorisierung: Bislang vernachlässigt; rechtliche Barrieren

- Zusatznutzen: Explizite Operationalisierung; unzureichende Transparenz, Begründung

- Überversorgung: Bislang vernachlässigt; Instanz?

\section{$\square$ Stellvertretende/Delegierte Werturteile}

- Verantwortliche Umsetzung = Einhaltung prozeduraler Werte

- Ziel: Vertrauen und Kooperationsbereitschaft

Abb. 8. Probleme bei der Wertefindung.

Ein weiterer Ansatz zu Beseitigung von Fehlversorgung ist das in den USA konzipierte und von Kanada übernommene Projekt «Choosing Wisely», das zur Erstellung von Top-5-Listen in Bezug auf Überversorgung geführt hat (Abb. 7). Die American Society of Clinical Oncology (ASCO) hat diesbezüglich eine Top-10-Liste erstellt. Derzeit sind 52 US-amerikanische und kanadische Fachgesellschaften in das Projekt eingebunden. Eine Studie berechnete, dass bei konsequenter Meidung der Maßnahmen, die in den ersten drei publizierten 


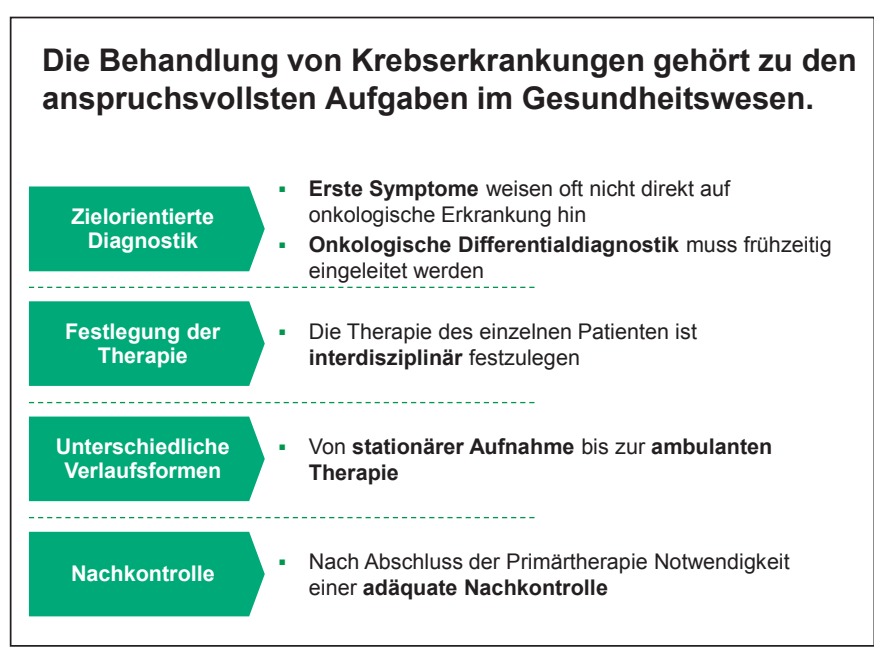

Abb. 9. Die Behandlung von Krebspatienten erfolgt interdisziplinär.

Top-5-Listen genannt wurden, eine Kosteneinsparung in den USA von 5 Milliarden US-Dollar erzielt werden kann [15].

Rationalisierung und Rationierung in der Medizin sind spätestens seit Abfassung des sogenannten «Ulmer Papiers» [16] im Jahr 2008 ein vieldiskutiertes Thema in der deutschen Ärzteschaft [17-19]. Um hier Entscheidungen zu treffen, müssen Institutionen und Gremien in der Lage sein, stellvertretende Werturteile für die Bevölkerung zu fällen. Die ethischen und praktischen Herausforderungen bestehen darin, diese «stellvertretenden Werturteile» transparent zu machen [20, 21] und zu operationalisieren (Abb. 8).

\section{Auflösung der sektoralen Versorgung als Steuerungsinstrument aus Sicht der Kostenträger}

\section{Referent: Uwe Deh, Berlin}

Komplexe Krankheitsbilder erfordern eine ausgefeilte Differentialdiagnostik und in der Regel eine interdisziplinäre Therapie. Eine bessere Verzahnung der medizinischen Angebote muss letztlich zum Ziel haben, die Versorgungsqualität aus Sicht der Patienten zu verbessern. Verzögert sich z.B. die Diagnosestellung - etwa aufgrund einer unspezifischen Symptomatik -, kann dies weitreichende Folgen für den weiteren Krankheitsverlauf haben. Ein für das Behandlungsergebnis entscheidendes Kriterium ist, ob der Patient binnen kurzer Frist die medizinisch notwendigen Diagnostik- und Behandlungsmaßnahmen erhält. Die Versorgung von Krebspatienten gehört unter diesem Aspekt zu den anspruchsvollsten Aufgaben im Gesundheitswesen (Abb. 9). Für onkologische Patienten sind Versorgungsprozesse auf dem aktuellen Stand des medizinischen Wissens prognoseentscheidend und damit lebensnotwendig: Der Weg führt von der sorgfältigen Anamnese und richtigen Diagnose über eine passende Therapie zu einem guten Behandlungsergebnis.
Qualitätssicherung muss essentieller Bestandteil onkologischer Versorgungsstrukturen sein.

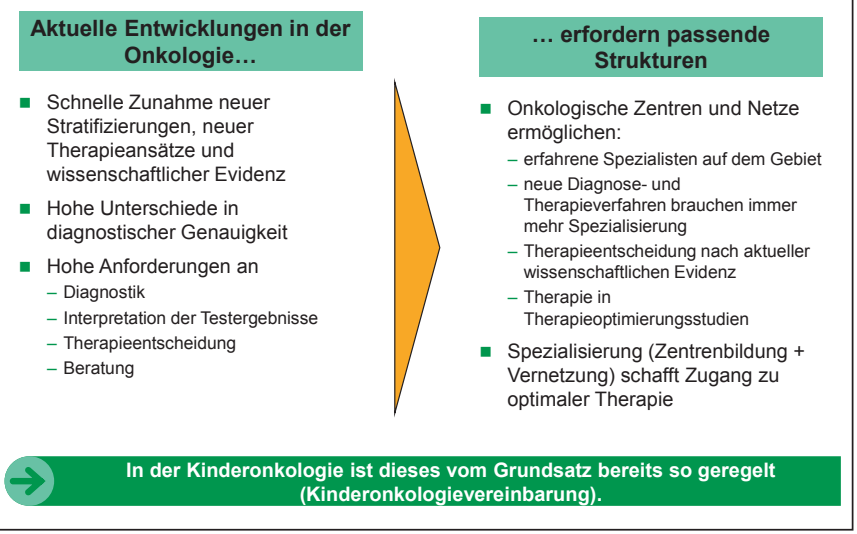

Abb. 10. Trend zu mehr onkologischen Zentren und höherer Spezialisierung.

\section{Anforderung an onkologische Versorgungsstrukturen}

Mit Blick auf die Onkologie stellt sich die Frage, wie die Versorgungsangebote durch Schaffung neuer Strukturen weiter verbessert werden können. Dabei geht es auch um einen beschleunigten Wissenstransfer, um etwa neue Therapien mit nachgewiesenem überlegenem Patientennutzen zeitnah in die flächendeckende Patientenversorgung zu bringen und unterlegene Diagnose- und Behandlungsmethoden schnell aufzugeben. Durch die Behandlung möglichst aller onkologischen Patienten in geeigneten Studien soll das notwendige Wissen zum Nutzen der Methoden gewonnen werden, und man sollte die Versorgung in die Zentren leiten, die die notwendige Qualifikation aufweisen. Dies mündet in die Forderung nach mehr Zentralisierung und Spezialisierung (Abb. 10), was eine erhebliche Umorganisation der bisherigen Behandlungsabläufe nach sich ziehen kann.

In der pädiatrischen Onkologie sind diese neuen Strukturen bereits gut etabliert und in der Vereinbarung zu Kinderonkologie entsprechend dokumentiert [22]. Auf Grundlage dieser Vereinbarung werden in Deutschland Kinder und Jugendliche mit hämatologisch-onkologischen Erkrankungen in Krankenhäusern seit 2007 nach verbindlichen Qualitätsstandards versorgt. Die Qualität der jeweils erforderlichen Behandlungen ist durch gezielte Zuweisungen in spezialisierte Krankenhäuser gewährleistet. Das Argument, solche Neuausrichtungen seien in Fachbereichen mit relativ geringer Patientenzahl einfacher vorzunehmen als in den großen Fachbereichen, sollte die Verhandlungspartner nicht von dem in der Kinderonkologie eingeschlagenen Weg abhalten.

\section{Status Quo der medizinischen Versorgungsstrukturen}

Die Strukturen im deutschen Gesundheitswesen behindern oft eine effiziente und effektive Versorgung der Patienten. Effektiv ist eine Behandlung dann, wenn sie dem aktuellen Wissenstand entspricht, effizient ist sie dann, wenn sie auch richtig durchgeführt wird. Hemmschuhe der Effizienz können unter 


\section{Strukturfragen der Versorgung}

1. Der beginnende Mangel an Hausärzten - Nicht bedarfsgerechter Spezialisierungsanreiz der Ärzteschaft

2. Der Run der Fachärzte aufs Massengeschäft, PKV und IGeL - Unzureichend definierter Versorgungsauftrag der Fachärzte

3. Die mangelnde Ambulantisierung spezialisierter Versorgungen - Trennung der Sektoren mit der Folge stationäre Leistungsvermehrung
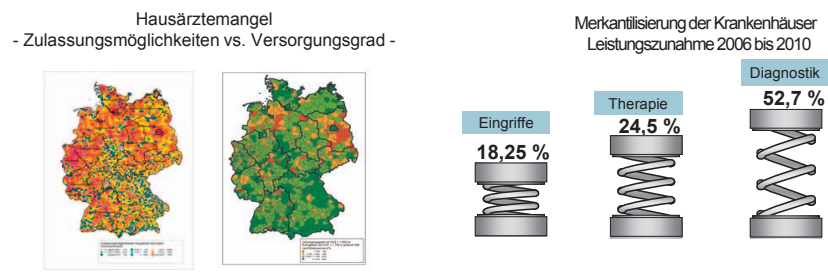

Abb. 11. Versorgungsprobleme im ambulanten Bereich.

anderem sein (in Analogie zum Managementbereich): ineffektive Prozesse, fehlerhafte Umsetzung und übermäßige Wartezeiten.

Eine Neustrukturierung im Gesundheitswesen mit Auflösung sektoraler Strukturen erfordert zunächst eine Bestandsaufnahme der heutigen Versorgungslandschaft. Sowohl im ambulanten Bereich (Abb. 11) wie auch in der stationären Versorgung (Abb. 12) müssen die gesellschaftlichen Entwicklungen (z.B. beginnender Mangel an Hausärzten) und gesundheitspolitischen Rahmenbedingungen (Krankenhausplanung) berücksichtigt werden. Gerade bei der Krankenhausplanung wurde bislang nicht erreicht, diese am tatsächlichen Bedarf und der notwendigen Qualität für den jeweiligen Versorgungsbereich auszurichten, was teilweise zu Überkapazitäten und Qualitätsdefiziten geführt hat. Wenn 78\% der Universitätskliniken kinderherzchirurgische Abteilungen haben, die dann aber nur zu durchschnittlich 59\% belegt sind, so kann an vielen Orten nicht die notwendige Erfahrung bei Operation und Nachbetreuung entstehen, die notwendig wäre. Dasselbe gilt z.B. auch für die im internationalen Vergleich viel zu hohe Zahl an Transplantationszentren in Deutschland, die dann um die Transplantation der begrenzten Zahl an Spenderorganen konkurrieren. In der derzeitigen Strukturdebatte geht es primär um die Verteilung von Ressourcen, sei es um den Einsatz von Humankapital oder die Verteilung der finanziellen Mittel, und viel zu wenig um eine optimale medizinische Versorgung der betroffenen Patienten.

Eine Überwindung sektoraler Strukturen durch eine bessere Zusammenarbeit zwischen Vertragsärzten und Krankenhäusern wird durch eine Vielzahl gesetzlicher Regelungen erschwert (Abb. 13). Sie fördern mehr die Sicherstellung partikulärer Interessen und weniger die Mittelverteilung im Sinne der Patienten. Auch die Vorgaben des G-BA zur ambulanten spezialfachärztlichen Versorgung (ASV) [23] sind nicht hinreichend präzise, da sie unter anderem keine klare Abgren-

\section{Strukturfragen stationär}

- Fortschritt: Produktivitätssprung der Krankenhäuser initiiert

$\rightarrow$ Trotz Zunahme der Morbidität hat sich die Zahl der Pflegetage verringert.

$\rightarrow$ Die KH-Mindestauslastung wird bei durchschnittlich $77 \%$ klar unterschritten

$\rightarrow$ Der zunehmende Anteil von Kurzliegern (38\%) zeigt weiteres Potential.

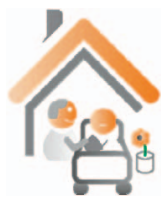

- Defizit: Betriebsgrößen, Spezialisierung und Ambulantisierung bleiben zurück

$\rightarrow 2 / 3$ der Häuser und 1/3 der Betten unterhalb der 300 Betten-Grenze

$\rightarrow$ Beispiel Lebentransplantationszentern: 24 in Deutschland, 9 im Vereinigten Königreich

$\rightarrow$ Beispiel Kinderherzchirurgie: $78 \%$ der Uniklinika bei Ø-Belegungsquote von $59 \%$

$\rightarrow$ Qualitätsvorteile der Spezialisierung bleiben weithin ungenutzt.

- Ursache: Bestätigende Krankenhausplanung erweist sich als überfordert

$\rightarrow$ Trotz Überkapazitäten Finanzierungsanteil der Länder unter 4,5\%.

$\rightarrow$ Verhandlungsanreiz für qualitätsorientierte Verträge fehlt (Kontrahierungszwang)

$\rightarrow$ Falsche Anreize und Zielvereinbarungen verschieben Indikationsgrenzen.

Abb. 12. Versorgungsstrukturen im stationären Bereich.

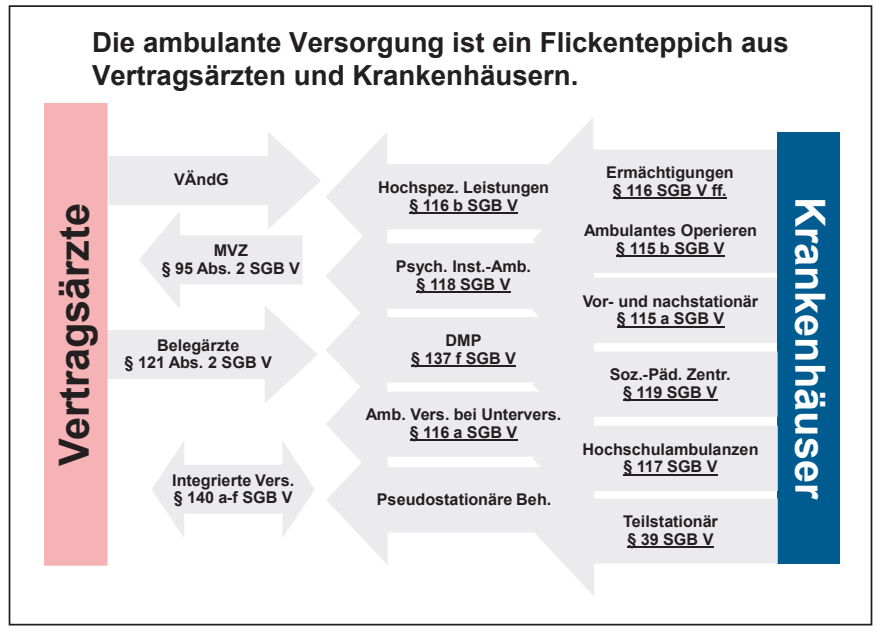

Abb. 13. Zahlreiche Verordnungen regeln das Miteinander von Vertragsärzten und Krankenhäusern.

zung der ASV-Leistungen zu anderen fachärztlichen Leistungen im niedergelassenen Bereich sowie zu stationären Leistungen erlauben. Statt eine Integration der Versorgungssektoren zu ermöglichen, fördert die aktuelle Fassung des $§ 116 \mathrm{~b}$ SGB V als gesetzliche Grundlage der ASV eher das Entstehen einer weiteren parallelen sektoralen Säule.

Hinsichtlich des Versorgungsauftrags scheinen die Steuerungsinstrumente des Systems im Feld zwischen Maximalund Primärversorgung zu versagen. Auf dieser Versorgungsebene tummeln sich neben Fachärzten und Krankenhäusern zahlreiche weitere Leistungserbringer wie Medizinische Versorgungszentren (MVZ), Arztnetze, Kooperationsgemeinschaften und Portalkliniken. Dies führt zu verschiedenen, für den Patienten schwerer zu durchschauenden und nicht aufeinander abgestimmten Konstrukten wie der spezialfachärztlichen Versorgung, sektorübergreifenden Versorgungskonzepten und der Verwendung der Zentrumsbezeichnung ohne zu- 


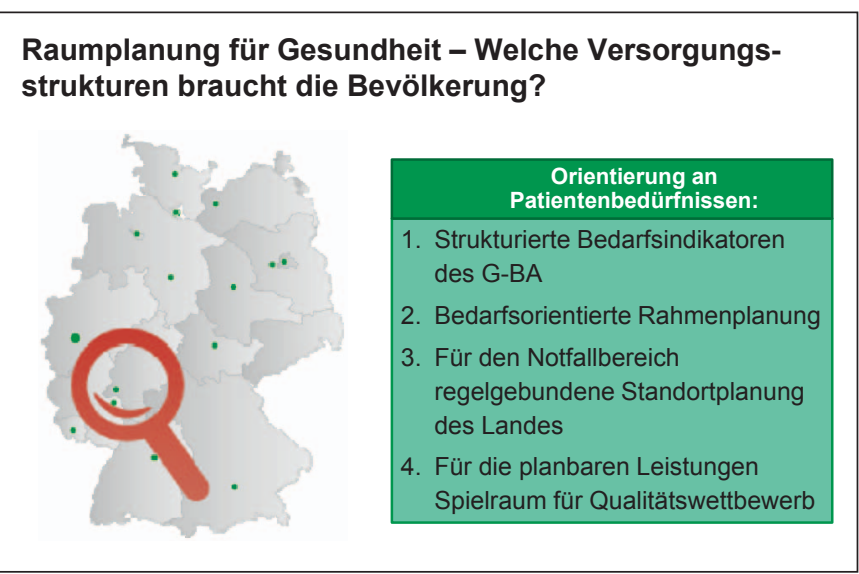

Abb. 14. Bedarfsorientierte Versorgungsplanung.

grundeliegende Qualitätsanforderung oder Zertifizierung. Bei all dem fehlt die Ausrichtung auf den Patienten im Sinne einer strukturierten abgestuften Versorgung.

In der Primärversorgung dagegen ist es einfacher, Versorgungsaufträge zu definieren. Die Ärzte übernehmen hier eine Lotsenfunktion, die relativ gut zu beschreiben ist. Aber auch im fachärztlichen Bereich sollte es möglich sein, den Versorgungsauftrag klarer als bisher herauszuarbeiten.

Die ASV als neue Versorgungsform entstand im Zuge der Neuregelung des § 116b SGB V durch das Versorgungsstrukturgesetz. Der Paragraph hat das eigentlich sinnvolle Ziel, dass Patienten mit seltenen und schwerwiegenden Erkrankungen durch besonders qualifizierte niedergelassene Fachärzte und Klinikärzte ambulant behandelt werden können. Diese Neuregelung greift allerdings zu kurz - auch in der onkologischen Versorgung. Die Vorgaben des Gesetzgebers für den Zugang der Leistungsbringer zu diesem neuen Versorgungsbereich vor allem hinsichtlich der Anforderungen an die Qualifikation, Strukturqualität und interdisziplinärere Zusammenarbeit sind so niedrig, dass die Qualität der Angebote für die betroffenen Patienten sich aller Voraussicht nach nicht verbessern wird und im Vergleich zu den bisherigen Strukturen - wie z.B. den onkologischen Schwerpunktpraxen - sogar deutlich unterschritten werden könnte. Mangels einer individuellen vertraglichen Ausgestaltung der ASV durch Leistungserbringer und Kostenträger fehlt den Krankenkassen die Möglichkeit, auf die Qualität dieser wichtigen Versorgung Einfluss zu nehmen.

\section{Wege zur Auflösung der sektoralen Versorgung}

Die ASV-Richtlinie wurde mangels entsprechender gesetzlicher Grundlage zudem ohne konkrete Bedarfsplanung auf den Weg gebracht, obwohl eine solche Planung für die verschiedenen Bereiche nötig ist und mit dem vorhandenen Datenmaterial auch zu realisieren wäre (Abb. 14).

Dabei geht es nicht darum, den Versorgungsbedarf anhand von vorhandenen Krankhausbetten, Arztsitzen oder Fallzahlen grob zu schätzen, sondern den Bedarf anhand der regional unterschiedlichen Bedürfnisse möglichst konkret zu ermitteln.

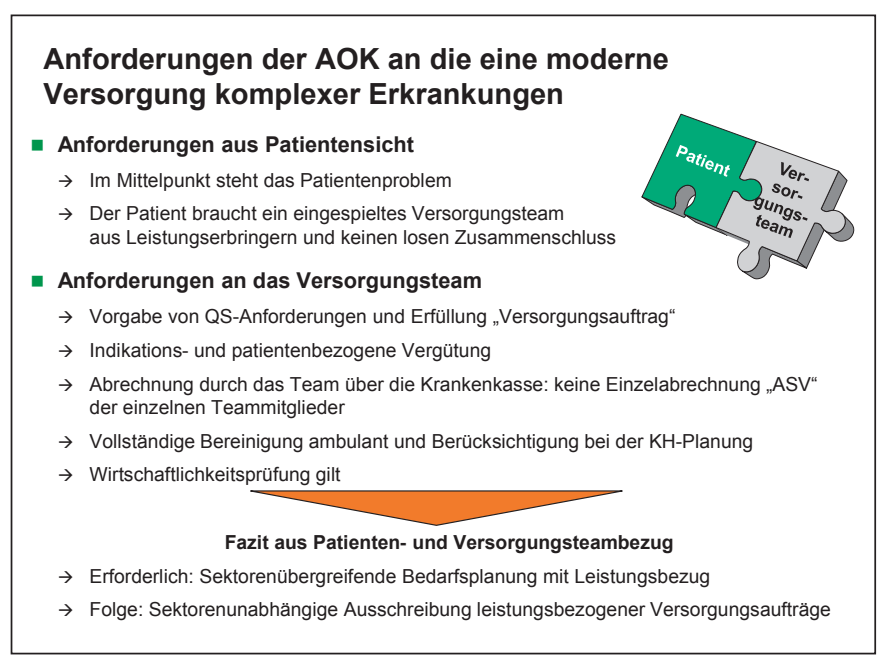

Abb. 15. Versorgung komplexer Erkrankungen aus Sicht des Kostenträgers.

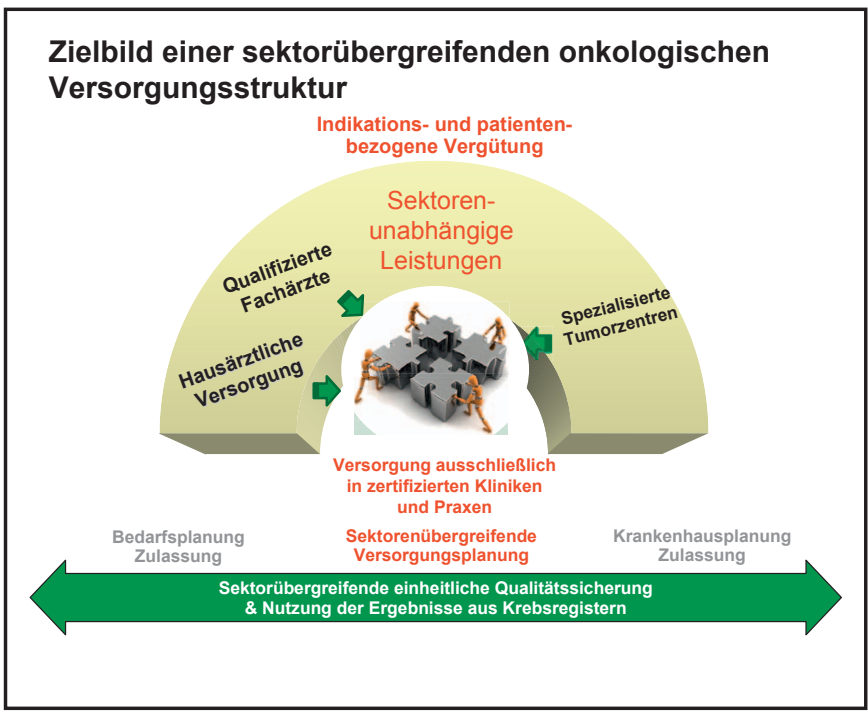

Abb. 16. Sektorenunabhängige Versorgung in der Onkologie.

Wünschenswert aus Sicht der Krankenkassen sind Versorgungsformen, bei denen Patient und Versorgungsteam eng zusammenarbeiten (Abb. 15). Es geht im Kern um eine Verbesserung der Rahmenbedingungen für eine teamorientierte Medizin. Die Möglichkeit, den jeweiligen regionalen Bedingungen entsprechende Qualitätsverträge (anstelle von Kollektivverträgen) abzuschließen, sollte genutzt werden.

Aus Sicht der AOK müsste sich eine moderne Krankenversorgung stärker an den Patienten hinsichtlich Indikation und Krankheitsepisode orientieren, um das Denken in sektoralen Strukturen aufzubrechen.

Noch steht anstelle des Teamgedankens eine mehr oder weniger starre Abgrenzung der Disziplinen und sektoralen Strukturen, was sich langfristig als Nachteil für die Qualität der Patientenversorgung und den Standort Deutschland als Arbeitsstätte für Mediziner erweisen könnte. Angehende 


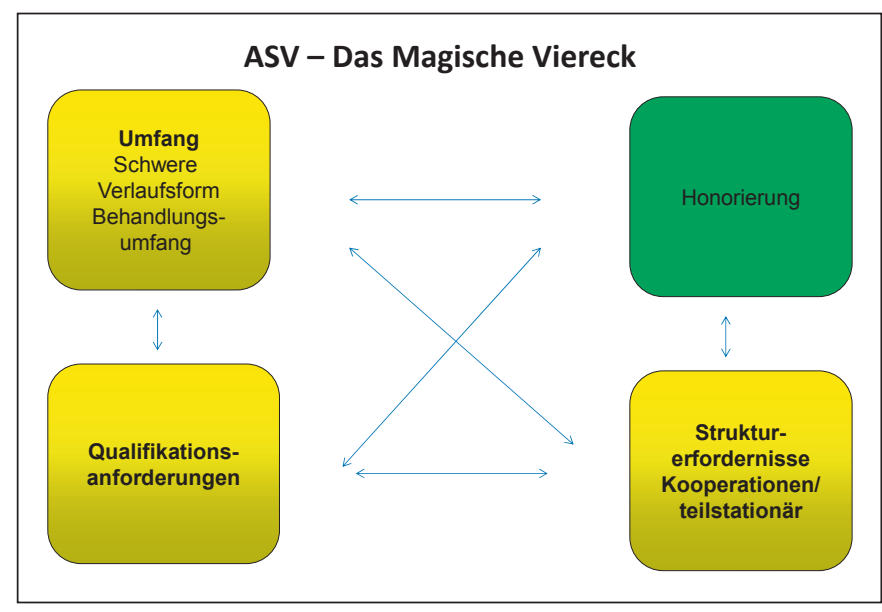

Abb. 17. Die ASV soll vielen Anforderungen gerecht werden.

Ärzte erwarten zeitgemäße Rahmenbedingungen, um eine moderne Patientenversorgung - wie in anderen Ländern längst üblich - praktizieren zu können.

Auch im onkologischen Bereich sollte künftig auf Grundlage indikations- und patientenbezogener Vergütungen die Erbringung sektorunabhängiger Leistungen im Vordergrund stehen (Abb. 16). Dafür müssen jedoch auch sektorübergreifende einheitliche angemessene Kriterien als Qualitätsmaßstab angelegt werden. Zudem sollten für die Beurteilung der Ergebnisqualität die Daten aus Krebsregistern stärker als bisher genutzt werden.

\section{Ist ASV eine langfristige Lösung?}

\section{Referent: Stephan Schmitz, Köln}

Mit Inkrafttreten des Gesetzes zur Verbesserung der Versorgungsstrukturen in der gesetzlichen Krankenversicherung (GKV-VStG) zum 1. Januar 2012 wurde die bisherige Regelung der ambulanten Behandlung im Krankenhaus in $\S 116 \mathrm{~b}$ SGB V durch die ASV ersetzt [24]. Sowohl die an der vertragsärztlichen Versorgung teilnehmende Leistungserbringer (niedergelassene Vertragsärztinnen und -ärzte sowie MVZ) als auch Krankenhäuser sollen künftig schwere Verlaufsformen von Erkrankungen mit besonderen Krankheitsverläufen, seltene Erkrankungen und Erkrankungszustände mit entsprechend geringen Fallzahlen sowie hochspezialisierte Leistungen unter grundsätzlich denselben Anforderungen ambulant behandeln können (Abb. 17).

Die Richtlinie zur ASV ist am 26. Juli 2014 in Kraft getreten, wobei in der Anlage die Behandlung von gastrointestinalen Tumoren und Tumoren der Bauchhöhle als erstes Beispiel für «schwere Verlaufsformen einer Erkrankung mit besonderem Krankheitsverlauf» konkretisiert wurde. Der Gesetzgeber hat die rechtlichen Voraussetzungen für die ASV geschaffen. Die Selbstverwaltung mit Kassenärztlicher Bundesverei-

\section{Versorgungsstrukturen}

1. Demografie mit zunehmenden Patientenzahlen - sozialen Anforderungen

2. Netzwerkstrukturen

- Innovationsnetzwerke

- Versorgungsnetzwerke pharmazeutische Kompetenz

- Krebszentren

3. Massive Veränderungsprozesse in der Organisation der ambulanten Leistungserbringung

Abb. 18. Veränderungen in der Versorgungsstruktur.

nigung (KBV), GKV-Spitzenverband und Deutscher Krankenhaus-Gesellschaft hat im G-BA die Richtlinie und die Anlagen verabschiedet. Die Anpassung des einheitlichen Bewertungsmaßstabs (EBM) an die neue Richtlinie erfolgte durch einen erweiterten Bewertungsausschuss für ärztliche Leistungen [25]. Nachdem die Voraussetzungen für den Start der ASV gastrointestinaler Tumorerkrankungen nunmehr erfüllt sind, liegt es an den Krankenhäusern und den Vertragsärzten, sie mit Leben zu erfüllen.

Die der ASV zugrundeliegende Idee besteht darin, dass Vertragsärzte und Krankenhäuser sektorübergreifend zu gleichen Bedingungen arbeiten können. Eine sektorenverbindende Zusammenarbeit von spezialisierten Fachärzten, Vertragsärzten und Krankenhäusern im Sinne eines dritten Sektors wurde auch vom Berufsverband der Niedergelassenen Hämatologen und Onkologen in Deutschland e.V. (BNHO) im Übrigen seit langem gefordert. Das hatte schließlich politische Entscheidungen zur Folge, die zunächst einmal als mutiger Schritt in Richtung eines Paradigmenwechsel zu begrüßen sind, nämlich weg von reiner Kostensenkung hin zu strukturellen Reformen.

Mit dem Inkrafttreten der ASV-RL werden sich die Perspektiven und die Positionierung der niedergelassenen spezialisierten Fachärzte weiter verändern. Hinzu kommen veränderte Versorgungsstrukturen (Abb. 18), die ebenfalls eine Weiterentwicklung der ambulanten Leistungserbringung erfordern.

Damit beschleunigt sich ein die Arbeit der niedergelassenen Hämatologen und Onkologen betreffender kontinuierlicher Veränderungsprozess. Dieser hat bereits 2004 mit Inkrafttreten des GKV-Modernisierungsgesetz (GMG) begonnen. Auf Ebene der Krankenhäuser führte die Einführung des neuen Klassifikationssystems Diagnosis Related Groups (DRGs) im Jahr 2003 zu vergleichbaren Umwälzungen. Es ist ein Trend zur Ambulantisierung der Medizin zu erkennen, die Öffnung der Krankenhäuser ist irreversibel und wird weiter zunehmen. Vorangetrieben werden die Änderungen ferner 


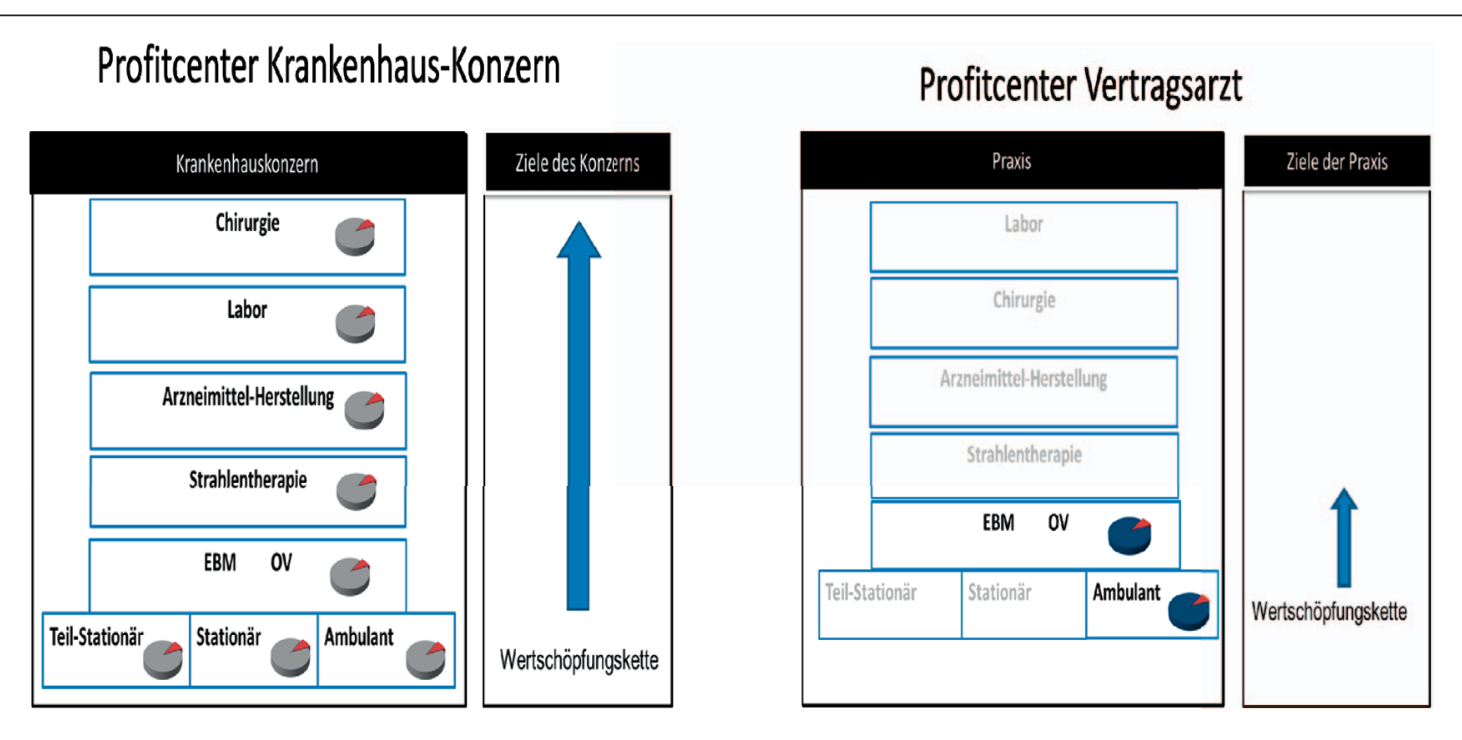

Abb. 19. Ungleichgewicht der Systeme.

durch die Ökonomisierung der Medizin und die Erwartungen der nachfolgenden Ärztegeneration an ihren Beruf. Für heutige angehende Ärzte steht laut Befragung von über 12000 Medizinstudenten durch die KBV die Vereinbarkeit von Familie und Beruf mit an vorderster Stelle [26]. Ein Großteil von ihnen - Männer wie Frauen - wünscht sich zudem die Möglichkeit von Teilzeitarbeit. Die angehenden Mediziner favorisieren bei der Niederlassung deutlich die Gemeinschaftspraxis gegenüber der Einzelpraxis - wenn sie denn überhaupt an eine Niederlassung denken. Dagegen sprechen laut Umfrage das hohe finanzielle Risiko, das hohe Maß an Bürokratie, eine unangemessene Honorierung sowie drohende Regressforderungen der Krankenkassen.

\section{Versorgungslage vor dem Jahr 2004}

Bis zum Jahr 2004 war die Situation für die Vertragsfachärzte relativ komfortabel. Die ambulante Behandlung der Patienten lag ausschließlich in den Händen der Vertragsärzte, im Gegenzug dafür garantierten die Vertragsärzte die Sicherstellung der Versorgung der Bevölkerung. Das System der klaren Abgrenzung zwischen ambulant und stationär wurde dann schrittweise aufgeweicht ( $\$ 140$, integrierte Versorgung, MVZ, $\S 116$ b alt, DMP). Daraus ergab sich ein ambulant-stationärer Grenzbereich, der durch zahlreiche Verordnungen reguliert wurde.

Nicht nur die niedergelassenen Ärzte, sondern auch die Kliniken haben in der Folge auf die veränderten Rahmenbedingungen reagiert. Die Krankenhäuser befinden sich seit Jahren im gegenseitigen Wettbewerb und agieren zunehmend - gefördert durch entsprechende Zusammenschlüsse und Aufkäufe - als Großkonzerne. Eine ähnliche Tendenz mit deutlichen Zeichen der Industrialisierung gibt es bei medizinischen Laboren, pathologischen Instituten und in Ansätzen auch im Bereich der Radiologie und Strahlentherapie.

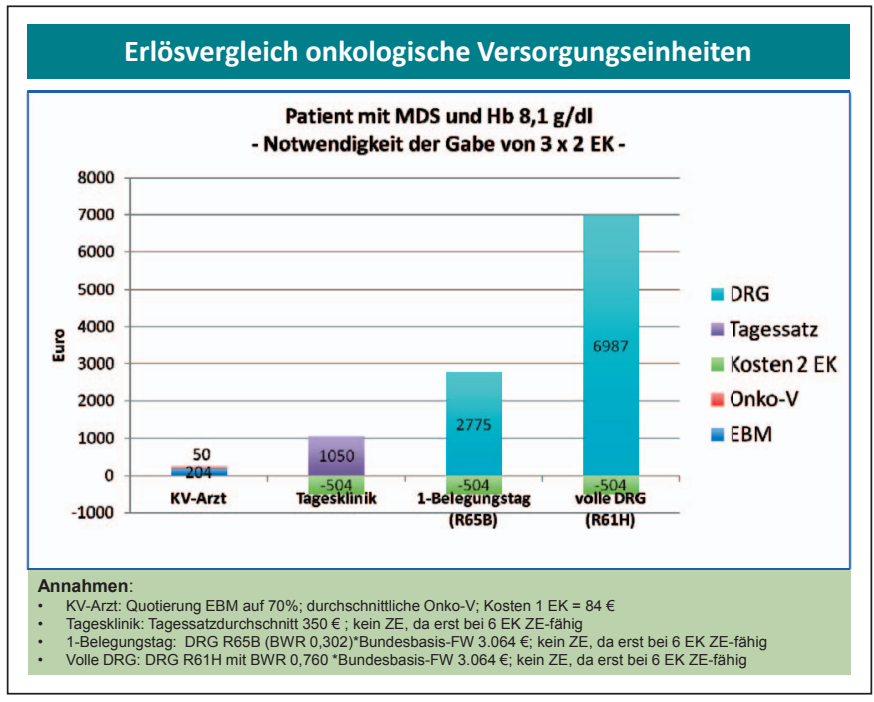

Abb. 20. Unterschiedliche Erlöse bei gleicher Indikation.

\section{Versorgungsituation heute}

Heute stehen sich zwei Versorgungssysteme gegenüber, die nicht mehr anhand der Aufgabenfelder - ambulante versus stationäre Versorgung - klar voneinander getrennt sind. Allerdings gelten für diese - politisch gewollte - Leistungsüberschneidung und -ausweitung unterschiedliche rechtliche Rahmenbedingungen.

Stellt man die beiden konkurrierenden Systeme aus rein ökonomischer Sicht als «Profitcenter» gegenüber, so ergibt sich für das System Krankenhaus durch die Aufnahme zusätzlicher ambulanter Angebote eine lukrative weitere Einnahmequelle im Rahmen der Wertschöpfungskette (Abb. 19). Die seit jeher auf ambulante Leistungen spezialisierten Fachärzte können ihr Angebot jedoch nicht in vergleichbarer Weise ausweiten. Im Bereich der Onkologie fallen die auf eine konkrete Leistung bezogenen Erlöse aufgrund der unterschiedlichen Abrechnungssysteme kleiner aus (Abb. 20), wobei insbeson- 
dere die Abrechnung über DRGs auch bei nur eintägiger stationärer Aufnahme für die Krankenhäuser vorteilhaft ist.

Festzuhalten bleibt, dass sich in den letzten 10 Jahren neben dem vertragsärztlichen Bereich ein weiterer ambulanter, an den Krankenhäusern angesiedelter Bereich fest etabliert hat. Es handelt sich somit um zwei grundverschiedene

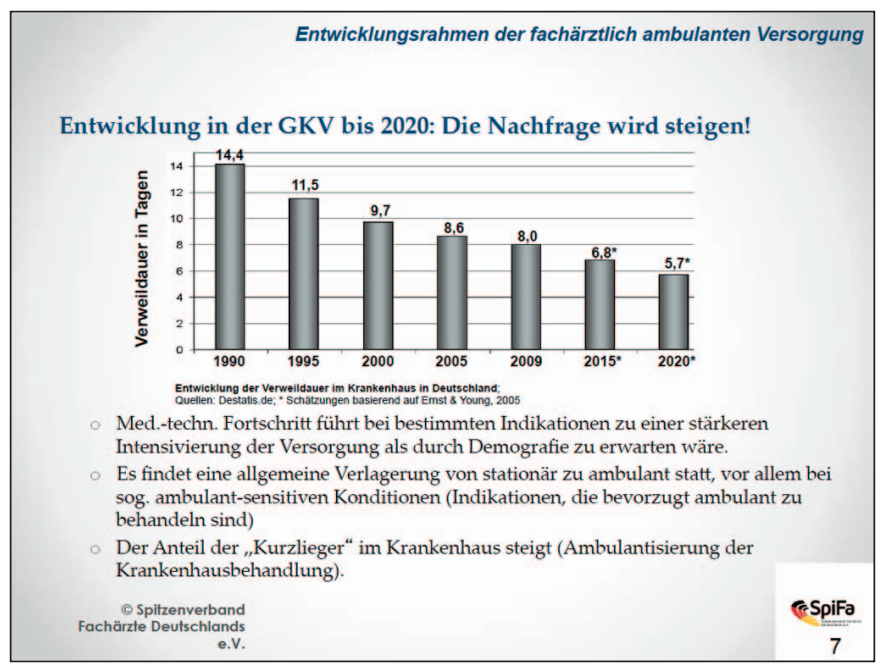

Abb. 21. Der Bedarf an ambulanter Versorgung wächst.
Systeme der Leistungserbringung, die, jedenfalls unter wettbewerbstheoretischen Gesichtspunkten, nicht miteinander kompatibel sind. Der Wettbewerb wird verzerrt, was zumindest als problematische Entwicklung diskutiert werden sollte.

Nach Einschätzung des Spitzenverbandes Fachärzte Deutschlands e.V. (SpiFa) wird es in den kommenden Jahren zu einer weiteren Ausdehnung ambulanter Leistungen kommen und damit einhergehend zu einer Verkürzung der stationären Verweildauer (Abb. 21).

Die verringerte Nachfrage im stationären Bereich dürfte zu Bettenabbau und Schließung einzelner Krankenhäuser führen. Dies wird besonders Kliniken der Grund- und Regelversorgung in Ballungsräumen und Krankenhäuser mit unwirtschaftlicher Arbeitsweise betreffen. Verschärft wird der Druck durch eine geplante monistische Krankenhausfinanzierung. Zudem wird man finanziell angeschlagenen Häusern als Ersatz für den Wegfall von Krankenhausbetten den institutionellen Zugang zur ambulanten Versorgung ermöglichen, was nur über eine Ausweitung der in $\S 116$ b SGB V geregelten ASV erfolgen kann.

\section{Fachärztliche Versorgung nimmt zu}

Trotz rückläufiger Bevölkerungszahl wird die fachärztliche Versorgung in den kommenden Jahren zunehmen. Nach An-

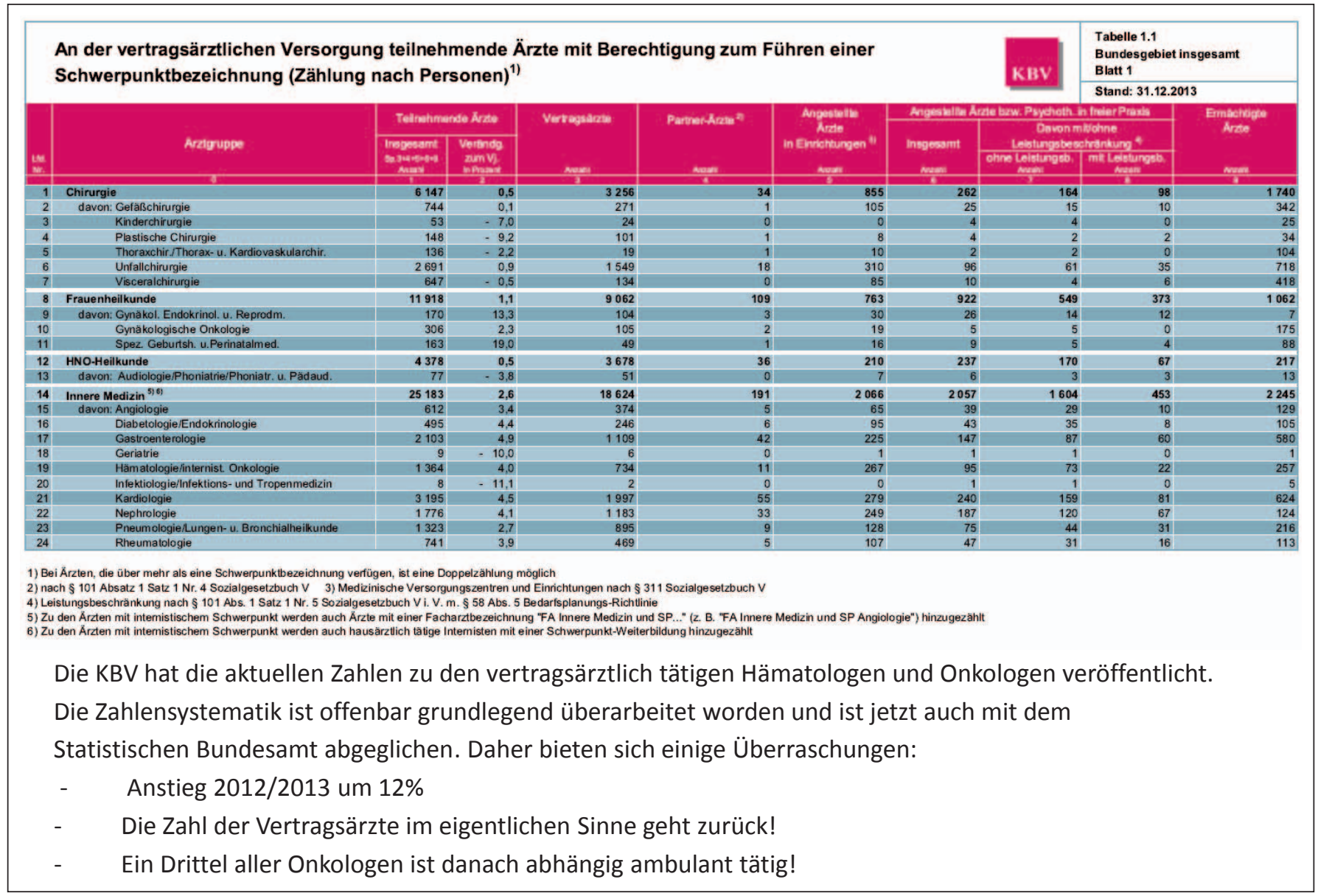

Abb. 22. Vertragsärztlich tätige Hämatologen und Onkologen. 


\section{Attribute der Freiberuflichkeit}
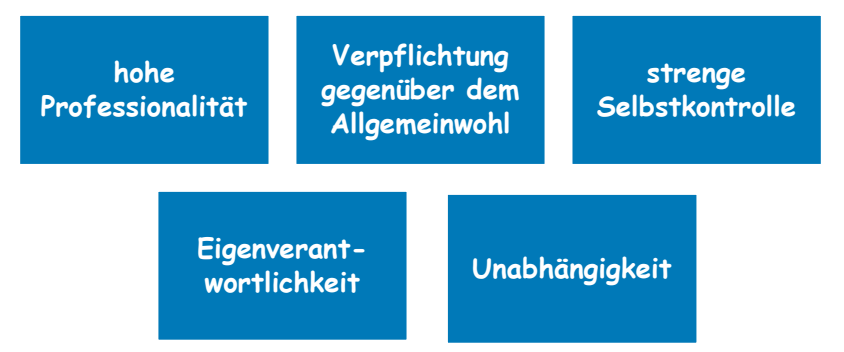

Abb. 23. Freiberuflichkeit im Spannungsfeld zwischen Anspruch und Wirklichkeit.

gabe des SpiFa dürfte der Anteil der Fachärzte am ambulanten Leistungsspektrum im Jahr 2020 bei über $70 \%$ liegen.

Die KBV hat aktuelle Daten zur vertragsärztlichen Versorgung durch Fachärzte mit Schwerpunktbezeichnung vorgelegt (Abb. 22). Ende 2013 waren insgesamt 1364 Hämatologen und Onkologen dieser Gruppe zuzuordnen. Ihre Zahl hat damit gegenüber den Vorjahren weiter zugenommen. Dabei ist die Zahl der nichtabhängig beschäftigten Vertragsärzte deutlich rückläufig, und die Zahl der in Praxen angestellten Vertragsärzte nimmt weiter zu. Dies spiegelt den Trend weg von der klassischen Freiberuflichkeit in eigener Praxis hin zu einer anderweitig organisierten Tätigkeit, sei es in Praxen oder Krankenhäusern.

Die weitere Öffnung der Krankenhäuser für den ambulanten Bereich unter dem Druck des Bettenabbaus wird diesen Megatrend noch verstärken. Sollte die ASV wie zu erwarten ausgedehnt werden und dann auch kurzstationäre Aufenthalte, die Versorgung in Tageskliniken, belegärztliche Behandlungen und anderes mehr umfassen, wird dies nicht ohne Auswirkungen auf den klassischen ambulanten, durch Vertragsärzte abgedeckten KV-Bereich bleiben. Dadurch wird sich das Rollenverständnis der Vertragsärzte ändern. Die Attribute der Freiberuflichkeit (Abb. 23). stehen zunehmend im Wettbewerb mit dem Primat der Wirtschaftlichkeit.

Die ASV ist vom Konzept her zu begrüßen, denn das Denken in sektoralen Strukturen ist nicht mehr zeitgemäß und ermöglich keine moderne Versorgung der Patienten. Es bleibt aber abzuwarten, wie der gute Ansatz nun weiterentwickelt wird. Mit der Schaffung des ASV-Bereichs wurden zwar ordnungspolitische Systeme - Krankenhäuser und freie Praxen zusammengeführt, was aber auch eine Änderung der Rahmenbedingungen und Neudefinierung der Rolle der Vertragsärzte erfordert.

Der BNHO begrüßt die Einführung des spezialfachärztlichen Sektors und die hohen Anforderungen an die interdisziplinäre Diagnostik und Therapie. Wir haben seit Jahren die Einrichtung eines dritten Sektors vorgeschlagen und die Entwicklung des Gesetzes begleitet. Trotzdem hängt die letztendliche Bewertung von der Umsetzung der Richtlinie vor Ort ab. Wenn die ASV Kooperationen zwischen Krankenhäusern und Vertragsärzten fördert, wird sie ein Erfolg. Wenn sie den Wettbewerb zwischen Krankenhäusern und Vertragsärzten weiter anheizt, wird sie die Versorgung nicht verbessern.

\section{Chancen und Risiken ökonomischer Steuerung aus Sicht der Leistungserbringer}

\section{Referentin: Irmtraut Gürkan, Heidelberg}

Die durchschnittliche Verweildauer der Patienten in deutschen Krankenhäusern ist in den vergangenen 20 Jahren um über $40 \%$ zurückgegangen. Parallel dazu wurde auch die Bettenzahl reduziert, allerdings nur um etwa $20 \%$. Das daraus resultierende regionale Überangebot an stationären Behandlungsplätzen hat bereits zahlreiche Kliniken in finanzielle Schwierigkeiten gebracht. Dies betrifft nicht nur Häuser der Grund- und Regelversorgung, sondern zunehmend auch Universitätskliniken mit maximalem Versorgungsangebot. Nach Schätzung des Verbands der Universitätsklinika Deutschland e.V. (VUD) werden im Jahr 2014 mehr als die Hälfte dieser Kliniken ein negatives Jahresergebnis mit Fehlbeträgen von über 1 Million EUR ausweisen. Ein Drittel der insgesamt 33 Universitätskliniken erwarten ein ausgeglichenes Jahresergebnis, und nur $13 \%$ werden voraussichtlich einen Überschuss erwirtschaften.

Die steigenden Kosten der Krankenhäuser, etwa aufgrund von teuren Tarifabschlüssen und gekürzten Veränderungsraten, können durch weitere Rationalisierungsmaßnahmen nicht mehr aufgefangen werden. Hinzu kommen neue Entwicklungen im Versorgungsbereich: So werden Patienten mit leichteren Krankheitsverläufen zunehmend ambulant behandelt, wobei dieser Trend auch durch die Krankenkassen durch vermehrte Überprüfung auf Fehlbelegung gefördert wird. Andererseits nimmt die Zahl aufwendiger Therapien im stationären Bereich zu (Komplexitätseffekt). Seit Einführung der DRGs versuchen die Kliniken gleichzeitig, durch immer weitere Rationalisierung die dem DRG-Erlös zugrunde liegenden Durchschnittskosten zu unterbieten, wodurch ein Kostensenkungswettbewerb entsteht (Kostendegressionseffekt).

Öffentlichkeitswirksame Berichte über Krankenhäuser, die trotz geringer Fallzahlen komplexe operative Eingriffe und hochspezialisierte Therapien anbieten, sowie internationale Vergleiche, wonach in Deutschland zu viel operiert wird, führen zu einem Vertrauensverlust in der Bevölkerung. Auf politischer Ebene gibt es allerdings nur zögerliche Versuche, solche Fehlanreize zu unterbinden. Hier sollten Ärzte und Fachgesellschaften von sich aus tätig werden, um ihren Qualitätsanspruch nach außen zu dokumentieren.

\section{Ökonomische Steuerungsinstrumente nutzen}

Eine Reihe von Möglichkeiten steht zur Verfügung, ökonomische Entwicklungen sinnvoll zu steuern und die vorhande- 


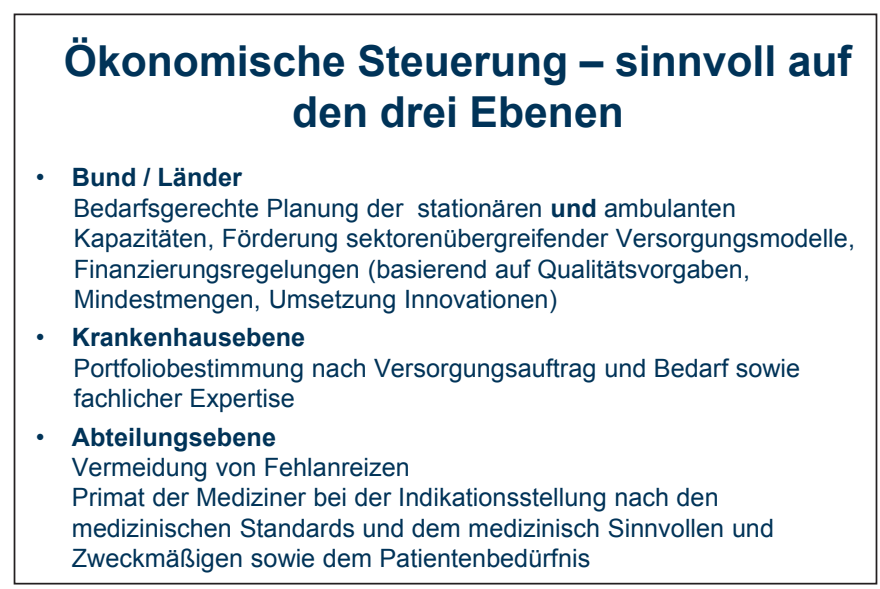

Abb. 24. Möglichkeiten der ökonomischen Steuerung.

\section{Fehlanreize bei Kostenerstattungen}

\section{- Ambulantisierung von eigentlich stationären} Leistungen wegen fehlender Erstattungsmöglichkeit:

$$
\begin{aligned}
& \text { - Innovationen bei fehlender Abbildung als NUB } \\
& \text { - Regional stark schwankende Finanzierung von } \\
& \text { NUB } \\
& \text { - Selbst bei Erstattung geplante Unterfinanzierung }
\end{aligned}
$$

Abb. 25. Mehr ambulante Versorgung aufgrund ökonomischer Fehlanreize?

nen Budgets in die gewünschte Richtung zu lenken (Abb. 24). Dies beginnt bei der bedarfsgerechten Planung durch Bund und Länder, die in Richtlinien zum Ausdruck kommt. Es wäre allerdings wünschenswert, wenn die Umsetzung der rechtlichen Vorgaben mit weniger bürokratischem Aufwand verbunden wäre. Eine löbliche Ausnahme sind hier z.B. die Ausführungsregelungen zum $\S 115$ b Abs. 1 SGB V - Ambulantes Operieren, die kurz und prägnant gefasst sind. Auf der Ebene der Krankenhausleitung ist es hilfreich, das Portfolio des Hauses eng am Versorgungsauftrag und der vorhandenen fachlichen Expertise auszulegen, statt die Leistungsausweitung an erste Stelle zu setzen. In den Krankenhausabteilungen sollten sich Steuerungsmaßnahmen vorrangig daran orientieren, was medizinisch sinnvoll und nötig ist. So reagierte das Universitätsklinikum Heidelberg bei Einführung der DRGs auf das damals noch wenig ausgereifte System nicht einfach mit einer Mengenausweitung, sondern hat auf medizinisch nötige Anpassungen der DRGs im Hinblick auf eine breitere Indikationsabdeckung gedrängt und diese auch durch intensive Mitarbeit in den Selbstverwaltungsgremien erreicht.

\section{Fehlanreize bei Kostenerstattungen}

\section{- Stationäre Behandlung von eigentlich ambulanten} Leistungen wegen fehlender Erstattungsmöglichkeit:

$$
\begin{aligned}
& \text { - PET (-CT) wird regional stationär durchgeführt } \\
& \text { - Teilfinanzierung über Tages-DRG } \\
& \text { - Teilweise Duldung durch Krankenkassen, obwohl } \\
& \text { primäre Fehlbelegung }
\end{aligned}
$$

Abb. 26. Stationäre Aufnahme bei PET-Untersuchungen.

\section{Noch keine Verzahnung}

- Die Werkzeuge sind alle vorhanden, aber...

- ...in einigen Ländern wurden (werden) ambulante Behandlungsmethoden nach §116b blockiert

- ...es herrscht lokal Konkurrenz zwischen Vertragsärzten und Krankenhäusern mit der Folge der Blockade

- ...komplett unterschiedliche Vergütungsmodelle im ambulante und stationären Sektor verhindern eine sinnvolle Zusammenarbeit

- ...Umsetzung ASV zu bürokratisch, zu zeitaufwendig

Abb. 27. Steuerungsinstrumente werden zu wenig genutzt.

Steuerungsmechanismen im Bereich Hämatologie und Onkologie

Die Hämatologie/Onkologie verfügt über zahlreiche Steuerungsmechanismen zur Patientenversorgung, die aber unzureichend genutzt werden. Daraus ergeben sich Fehlanreize bei Kostenerstattungen (Abb. 25), die zu einer Verlagerung originär stationärer Leistungen in den ambulanten Bereich führen können. Dazu tragen auch Unsicherheiten bei der Finanzierung neuer Untersuchungs- und Behandlungsmethoden (NUB) im stationären Sektor bei. Dies gilt allemal für Innovationen, die nicht als NUB anerkannt sind. Aber auch nach Klassifizierung einer NUB als erstattungsfähige Maßnahme können die dafür anfallenden Kosten oft erst zeitverzögert und in regional unterschiedlicher Höhe abgerechnet werden, was zwangsläufig zu einer Unterfinanzierung in diesem Bereich führt.

Im Gegenzug werden aber auch ambulant zu versorgende Patienten gelegentlich stationär aufgenommen, da die Leistungen anderweitig nicht abzurechnen sind. Dies gilt etwa in bestimmten Regionen für die Durchführung von PET-Untersuchungen (Abb. 26). 


\section{Fazit aus Sicht eines Uniklinikums}

- Ambulante Leistungen sind längst auch für Universitätskliniken strategisch bedeutsam und relevante Umsatzbringer

- Rekrutierung stationärer und teilstationärer Patienten über die Ambulanzen

- Ersatz für wegbrechende Vergütungen im stationären Bereich (klassisch im Bereich der Augenheilkunde)

- Eigenständige Leistungen im Sinne von „ambulanter Maximalversorgung“ (Beispiele: Stammzellentnahme, Stammzelltransplantation)

Abb. 28. Ambulante Leistungen gehören zum Spektrum der Krankenhäuser.

\section{Grundkonsens zur Kooperation in unserer Region}

- Wahrnehmung des Versorgungsauftrages im stationären Sektor entsprechend der Versorgungsstufe (Grund-, Regel-, Maximalversorgung)

- Abstimmung / Verträge über Behandlungsspektren Patientenzuweisungen und Abverlegungen

- Uniklinikum: Letztinstanz in der Behandlungskette (Diagnostik, Therapie), nicht in der Phase der „Ausbehandlung“

- Abstimmung über stationäre / teilstationäre / ambulante (Folge-)Behandlung mit Schwerpunktpraxen

- Komplexe ambulante Fälle in Spezialambulanzen des Uniklinikums (Transplantationspatienten, Mukoviszidosepatienten, schwere Stoffwechselerkrankungen)

Abb. 29. Kooperationen des Universitätsklinikums Heidelberg.

Es ist bedauerlich, dass die vorhandenen Instrumente bisher nicht wesentlich zu einer verbesserten Zusammenarbeit zwischen Vertragsärzten und Krankenhäusern geführt haben, was auch mit der Unvereinbarkeit der beiden Systeme, etwa im Hinblick auf die Vergütung der Leistungen, zu tun hat (Abb. 27). Mit Einführung der ASV dürfte sich daran vorerst nicht viel ändern, da die Umsetzung sehr zeitaufwendig ist und nur schrittweise erfolgt. Ende 2014 sind gerade einmal die gastrointestinalen Tumoren geregelt.

Ambulante Leistungen sind für Krankenhäuser ein wichtiges Standbein und nicht mehr aus dem Leistungsspektrum wegzudenken (Abb. 28). Der (unter Umständen wiederholte) Wechsel von der stationären zur ambulanten Versorgung entspricht in verschiedenen Indikationen dem medizinischen Standard und steht dem Patienten als Angebot zumindest im Rahmen der Maximalversorgung durch Universitätskliniken zur Verfügung.

Für Häuser mit Ausbildungsauftrag, z.B. für die Weiterbildung zum Augenarzt, ist es unabdingbar, auch ambulante Leistungen erbringen zu können. Das Universitätsklinikum

\section{Prädiktive, molekularpathologische Diagnostik - Vereinbarung UKHD}

- Ziel: Identifizierung derjenigen Patienten, die die Chance haben, auf bestimmte Therapien (Medikamente) anzusprechen

- Die Panel-Sequenzierung wird in hohem Maße für ambulante Patienten erbracht und ist über die Ambulanzpauschale nicht refinanzierbar

- Vereinbarung definierter molekular-pathologischer Diagnostik im Rahmen der Hochschulambulanzvergütung ab 2013 nach Begutachtung durch den MDK für verschiedene Tumorentitäten (u.a. malignes Melanom), Obergrenze ca. $660 \mathrm{~T} / €$

Abb. 30. Diagnostik-Vereinbarung am Universitätsklinikum Heidel berg.

\section{Fazit}

- Der medizinische Fortschritt, Innovationen und Prozessverbesserungen in der Patientenbehandlung ermöglichen die Ambulantisierung ehemals stationär erbrachter Leistungen. Dieser Megatrend gilt ganz besonders für die Onkologie und Hämatologie (immer weniger Betten, dafür große Tageskliniken, Ambulanzeinheiten)

- Dabei müssen weiterhin spezielle Fachkenntnisse und interdisziplinäre Diagnostik und Behandlung zum Einsatz kommen

- Die Durchlässigkeit der Sektorengrenzen, in fast allen Ländern gelebt, muss auch in Deutschland umgesetzt werden, einschließlich ausreichender Finanzierungsregelungen

- Ob dies mit der ASV in endlicher Zeit gelingt, ist fraglich

- Bedarfsorientierte Regelungen, mit anderen Leistungserbringern vor Ort und im Einvernehmen mit den örtlichen Kostenträgern, erscheinen derzeit zielführender

Abb. 31. Trends in der Versorgung.

Heidelberg hatte im Jahr 2013 einen Gesamtumsatz von 925 Millionen EUR, von dem knapp 14\% (128 Millionen EUR) durch ambulante Erlöse erzielt wurde.

Erfahrungen mit ambulanter Versorgung am Universitätsklinikum Heidelberg

Am Universitätsklinikum Heidelberg gibt es verschiedene Versorgungs- und Abrechnungsmodelle, die geordnet und nebeneinander angewendet werden. Dazu gehören neben der Abrechnung über DRGs auch teilstationäre Tagespauschalen, Abrechnungsmodalitäten nach § 116b (ASV), § 117 (Hochschulambulanzvergütung), § 118 (PIA) und $§ 119$ (Sozialpädiatrisches Zentrum) nach SGB V und andere. Entscheidend ist auch eine transparente Abgrenzung dieser verschiedenen Vergütungsmöglichkeiten gegeneinander, um «Rosinenpickerei» zu verhindern und damit bei den Kostenträgern Vertrauen zu schaffen.

Ferner gibt es in der Heidelberger Region einen Grundkonsens (Abb. 29), der weitere Vereinbarungen zur Erbrin- 
gung innovativer Leistungen und zur Schaffung neuer Behandlungsstrukturen auf lokaler Ebene ermöglicht.

Ein Beispiel für neue Vergütungsmodelle ist die im Jahr 2012 geschlossene Vereinbarung zwischen dem Universitätsklinikum Heidelberg und den Kostenträgern in Bezug auf die ambulante Stammzelltransplantation. Die Vergütung erfolgt als ambulante DRG-Leistung tagesbezogen bis $\mathrm{zu}$ einem Deckelbetrag zuzüglich einem Zusatzentgelt (ZE). Muss der Patient stationär weiterbehandelt werden, ist eine entsprechend DRG-Abrechnung ebenfalls möglich, was gewissermaßen einer Teilung des finanziellen Risikos zwischen Kasse und Krankhaus gleichkommt. Bisher wurden 12 Patienten ambulant autolog transplantiert, von denen 9 ausschließlich ambulant versorgt werden konnten.

\section{Implementierung neuer diagnostischer Verfahren in} Vergütungsmodelle

Da der Einsatz neuer Tools im Bereich der prädiktiven, molekularpathologischen Diagnostik nicht innerhalb des DRG-Systems abgebildet ist, sind die Vergütungsmodalitäten im stationären Bereich unklar. Die Diagnostik ist zwar darstellbar über Codes im Operationen- und Prozedurenschlüssel, was aber für die Vergütung nicht relevant ist. Da die Kassen eine enorme Leistungsausweitung in diesem Bereich befürchten, stehen neue, voraussichtlich sektorübergreifende Vereinbarungen noch aus. Zu klären ist dabei, für welche Patienten eine komplexe Diagnostik überhaupt nötig ist und ob im Falle einer Beschränkung auf bestimmte Entitäten eine gesonderte Vergütung nötig ist. Auch stellt sich die Frage, ob die neue Diagnostik indikationsunabhängig über eine Pauschale vergütet werden sollte und inwieweit sie sektorunabhängig organisiert werden kann, um Verschiebungsanreize zu vermeiden. Derzeit wird von den Kostenträgern eine Lösung favorisiert, die eine sektorunabhängige Erstattung über das pathologische Institut z.B. im Rahmen eine Vertrages zur integrierten Versorgung vorsieht, was allerdings eine zusätzliche Qualitätssicherung und eine exakte Leistungsbeschreibung nötig macht.

Zwischenzeitlich hat das Universitätsklinikum Heidelberg für die Hochschulambulanz mit den Kassen einen gedeckelten Erstattungsrahmen für definierte Diagnostikverfahren vertraglich vereinbart (Abb. 30). Die jetzt anstehende Evaluierung wird zeigen, ob dieses vor 2 Jahren eingeführte Vergütungsmodell für beide Seiten funktioniert.

Wir müssen im Spannungsfeld zwischen stationärer und ambulanter Versorgung Lösungen finden, die medizinisch sinnvoll, für den Patienten von Vorteil und zudem finanzierbar sind (Abb. 31). Die Ambulantisierung ehemals stationärer Leistungen sollte nicht durch finanzielle Restriktionen behindert werden; die Durchlässigkeit der Sektorengrenzen muss weiter gefördert werden. Ob diese Ziele zeitnah mit dem Instrument ASV erreicht werden können, ist fraglich. Vorerst werden bedarfsorientierte Regelungen vor Ort nötig sein - in Einzelfällen auch in Form von Selektivverträgen und Modellvorhaben.

\section{DRG FALLPAUSCHALE KOSTEN UND ERLÖSE}

$$
\begin{aligned}
& \text { - Kosten } \\
& \text { = Personal } \\
& \text { " Infrastruktur } \\
& \text { " Sachkosten } \\
& \text { " Medikamente } \\
& \text { - ZE } \\
& \text { " NUB } \\
& \text { " Rest } \\
& \text { " Externe Leistungen } \\
& \text { " ILV }
\end{aligned}
$$

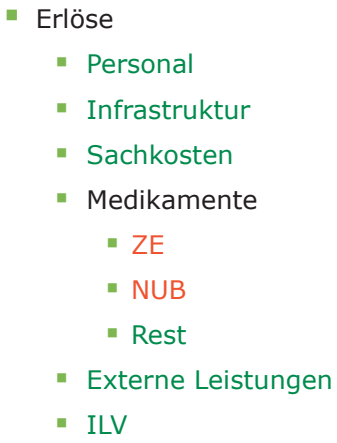

Abb. 32. DRG-System: einfließende Kosten und Erlöse.

\section{Bedeutung der Bewertung und Aufteilung von DRGs für die innerhalb des Krankenhauses stattfindende Budgetierung und Aufteilung der Ressourcen}

\section{Referent: Helmut Ostermann, München}

Immer mehr Kliniken können nicht mehr ökonomisch arbeiten, da die im Haus entstehenden Kosten die erzielten Erlöse dauerhaft übersteigen. Um die wirtschaftlichen Schwachstellen aufzudecken, ist eine genauere Betrachtung der Erlösverteilung auf Abteilungsebene zweifellos hilfreich. Dabei ist es zunächst am einfachsten, die Abteilungen und Kliniken anhand ihrer finanziellen Gewinne beziehungsweise Verluste zu vergleichen. Allerdings ist es fraglich, ob die bloße Gegenüberstellung von «gut» und «schlecht» wirtschaftenden Abteilungen sinnvoll ist. Eine «gut» wirtschaftende Klinikabteilung wird aus eben diesem Grund um mehr Personal und bessere Ausstattung zwecks weiterer Erlössteigerung anfragen, aber auch einer «schlecht» wirtschaftenden Abteilung wird man kaum finanzielle Mittel und Personal entziehen können. Vergleiche allein auf Basis von Ergebnisrechnungen sind deshalb aus unserer Sicht nicht zielführend. Die Wirtschaftlichkeit der Abteilungen lässt sich eher daran ablesen, ob die jeweils angestrebte Leistungsmenge im Vergleichszeitraum erzielt worden ist.

\section{Kliniken sind keine «Profitcenter»}

Dabei ist zu berücksichtigen, dass die einzelnen Kliniken eines Hauses ihre Kosten nur über die Ausweitung der Leistungsmenge, nicht aber durch Drehen an der Preisschraube senken können. Eine Klinikabteilung ist eben kein «Profitcenter», da sie z.B. mit externen Leistungserbringern wie Laboren und Zulieferern nicht eigenständig Preise aushandeln kann.

Die Kosten einer Abteilung setzen sich zusammen aus Personal- und Sachkosten, Medikamentenkosten einschließlich ZE und Entgelten für NUB sowie Kosten für externe Leistungen. Hinzu kommt der Aufwand für die nötige Infra- 


\section{DER STATIONÄRE ERLÖS}

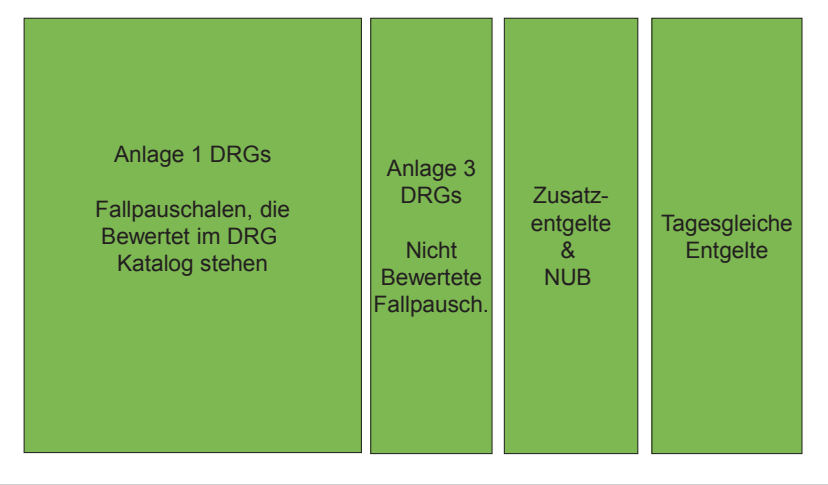

Abb. 33. Aufteilung der stationären Erlöse.

struktur (Transportdienst, Verwaltung, Energie und Instandhaltung), die als wenig zu beeinflussende Fixkosten zu Buche schlagen. Die Abbildung der Kosten und Erlöse erfolgt mit Ausnahme von ZE und NUB über die DRG-Fallpauschalen (Abb. 32). Auf der Erlösseite sind entsprechend dem Leistungsangebot der Abteilungen weitere stationär erzielte Einnahmen zu erfassen (Abb. 33).

\section{Verlegung innerhalb des Hauses - wie teilt sich der Erlös auf?}

Um ein Gesamtbild der Wirtschaftlichkeit eines Krankenhauses und seiner einzelnen Fachabteilungen zu erhalten, ist eine Aufschlüsselung der stationären Erlöse sinnvoll. Für die Zuweisung der Erlöse bei hausinterner Verlegung gibt es verschiedene Ansätze, wie sich am Beispiel eines fiktiven Patienten mit einer Fallschwere von 2,0 Case-Mix(CM)-Punkten und einer stationären Verweildauer von 10 Tagen zeigen lässt. Wird für die Erlösaufteilung eine Zeitpunktsicht gewählt, erhält die den Patienten entlassende Abteilung alle CM-Punkte, wodurch Abteilungen mit hohem Verlegungsanteil durchgehend im Nachteil sind. Wird für die Aufteilung dagegen eine Zeitraumsicht gewählt, erhält jede Abteilung für die Zeit, die der Patient dort verbracht hat, anteilig die zugehörige DRGFallpauschale. Bleibt der gewählte Beispielpatient auf insgesamt fünf Stationen für jeweils 2 Tage, erhält also jede Station 0,4 CM-Punkte. Allerdings machen Fälle mit hohen CM-Werten die Statistik kaum mehr interpretierbar. Zudem sollte die Gruppe der besonders kostenaufwendigen Prä-MDCs (MDC = Major Diagnostic Category) gesondert betrachtet werden.

Die Erlösaufteilung nach Zeitraumsicht ist demnach nur sinnvoll, wenn der Aufwand der einzelnen Abteilungen in etwa gleich ist, was aber eher selten der Fall ist. Wird mit einer aufwandkorrigierten Zeitraumsicht gerechnet, ist zunächst ein Abteilungs-CM für alle nichtverlegten Patienten zu ermitteln. Durch Multiplikation dieses Werts mit der tatsächlichen Verweildauer des jeweiligen Patienten ergibt sich eine korrigierte Verweildauer als Berechnungsgrundlage für die anteiligen

\section{ERLÖSAUFTEILUNG}

- Erlösaufteilung nach Verweildauer

- Jede Verweildauerminuten wird gleich hoch gewichtet

- Erlösaufteilung nach gewichteter Verweildauer

- Üblicher Weise wird der Abteilungs-CMI als Gewichtung verwendet

- Eine Minute in der Chirurgie-Abteilung mit CMI 1,0 ist nur halb so «teuer» wie eine Minute in der Inneren-Abteilung mit CMI 2,0

- Erlösaufteilung nach INEK-Matrix

- Komplexe Aufteilungsprinzipien

Abb. 34. Möglichkeiten der Erlösaufteilung.

ERLÖSAUFTEILUNG - PROBLEME
- Unterschiede im Kostenaufwand (Personal / Sachkosten)
zwischen Abteilungen sind schwer abzubilden
- Nicht Bettenführende Abteilungen müssen aus den DRG
Erlösen finanziert werden
- ILV-Abbildung weiterhin schwierig
- F \& L-Zuordnung bei Kosten / Erlösen schwierig

Abb. 35. Probleme bei der Erlösaufteilung.

CM-Punkte der beteiligten Fachrichtungen. Auf diese Weise werden Abteilungen mit hohem Kostenaufwand (wie die Anästhesie) gegenüber solchen mit geringerem Kostenaufwand (z.B. Palliativmedizin) bei gleicher Verweildauer des Patienten finanziell begünstigt.

\section{Ergebnisberechnung bleibt unscharf}

Ein Vergleich der Wirtschaftlichkeit der verschiedenen Abteilungen auf Basis solcher Ergebnisberechnungen ist nicht der Weisheit letzter Schluss, denn letztlich bleibt von den Erlösen nach Abzug von Primärkosten, Interner Leistungsverrechnung (ILV) und Gemeinkostenumlage ein positives oder negatives «Endergebnis» übrig, an dem sich die Abteilungen messen. Um solche pauschalen Beurteilungen zu vermeiden, werden in unserem Haus die Kostenarten ILV und Gemeinkosten nicht mehr auf Abteilungsebene kommuniziert, sondern stattdessen die Leistungserbringung für Vergleichszwecke gewählt.

Auch bleibt die Ergebnisrechnung anhand von Erlösen und Kosten trotz detaillierter DRG-Aufschlüsselung und ZE- 
Abb. 36. In der InEK-Matrix abgebildete Kostenstellen.

\section{WIE KANN DIE MATRIX GELESEN WERDEN ... KOSTENSTELLEN}
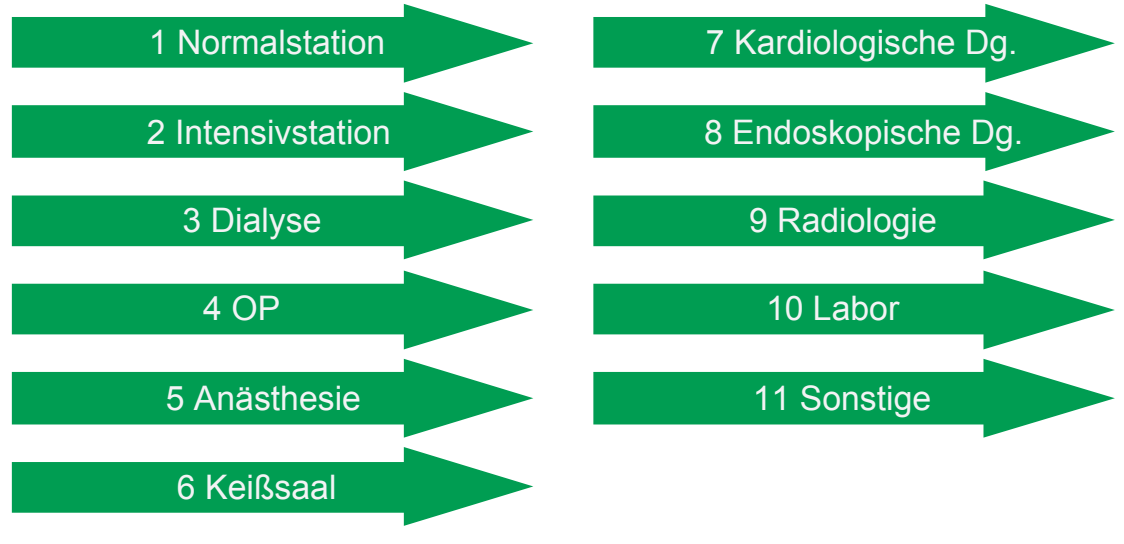

TRENNUNGSRECHNUNG (VEREINFACHTE DARSTELLUNG)
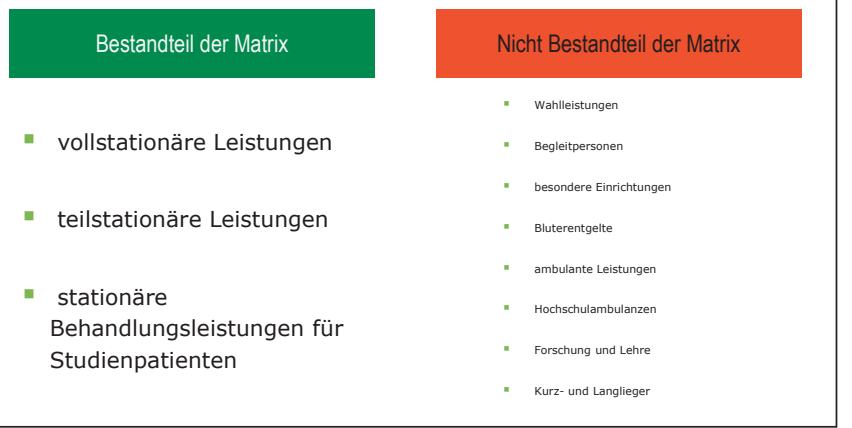

Abb. 37. Nicht alle Leistungen werden von der InEK-Matrix erfasst.

Regelungen bis zu einem gewissen Grad ungenau, weil Variablen wie die ILV miteinbezogen werden müssen. Die korrekte Ermittlung des Aufwands der einzelnen Abteilungen mit interner Verrechnung der Erlöse einerseits und den für Dritte erbrachten Leistungen andererseits ist eine schwierige Aufgabe. Bei interdisziplinärer Patientenversorgung benachteiligt eine solche Erlösaufteilung stets den Anforderer einer internen Leistung und begünstigt auf der anderen Seite die entlassende Einrichtung hinsichtlich der Abrechnung von mehrfach erbrachten ZE und NUB.

Die vorhandenen Modelle zur Erlösaufteilung sind folglich aus verschiedenen Gründen problembehaftet (Abb. 34, 35).

\section{Arbeiten mit der InEK-Kalkulationsmatrix}

Das Institut für das Entgeltsystem im Krankenhaus GmbH (InEK) wurde 2001 von Spitzenverbänden der gesetzlichen und privaten Krankenkassen gemeinsam mit der Deutschen Krankenhausgesellschaft eingerichtet, um das DRG-Fallpau- schalensystem fortlaufend an die in den Krankenhäusern anfallenden Durchschnittskosten anzupassen. Das InEK stützt sich dabei auf die Daten der sogenannten Kalkulationskrankenhäuser, die für die einzelnen DRG-Fälle die damit verbunden Kosten, unterteilt nach Kostenarten und Kostenstellen, ermitteln. Aus den Daten, die dem InEK von diesen Häusern zur Verfügung gestellt werden, errechnet das Institut für jede DRG die durchschnittlichen Kosten und setzt das jeweilige Relativgewicht fest.

Die InEK-Matrix ist zweidimensional aufgebaut mit einer Untergliederung der Kostenarten und Kostenstellen (Abb. 36). Aus der Matrix lässt sich demnach für jede einzelne DRG entnehmen, welche Kosten in welchem Bereich durchschnittlich anfallen und dementsprechend über die DRG vergütet werden. Durch Vergleich der eigenen Kosten mit den DRGNormkosten wird dann ersichtlich, in welchen Bereichen DRG-kostendeckend gearbeitet wird und in welchen nicht.

Bei der Erlösberechnung mit Hilfe der InEK-Matrix ist zu beachten, dass nicht alle von einem Krankenhaus potentiell angebotenen Leistungen damit erfasst werden, sondern nur eine Berechnung der voll- und teilstationären Leistungen möglich ist (Abb. 37).

\section{Beispiel für das Befüllen der Matrix}

Für relativ einfache DRG-Fallpauschalen wie die «vaginale Entbindung ohne komplizierende Diagnose» ist die Kostenverteilung in der Regel gut nachvollziehbar. So entfallen für die Entbindung 94\% der Kosten auf die Normalstation und den Kreißsaal und verbleiben so weitgehend in der Gynäkologie. Die Kosten entstehen vor allem im Bereich Personal (58\%) und medizinische Infrastruktur (28\%).

Bei schwierigeren Fällen etwa im Bereich der allogenen Knochenmarktransplantation (DRG A04C) machen die Kosten für Intensivstation und Labor zusammen mehr als 90\% der 


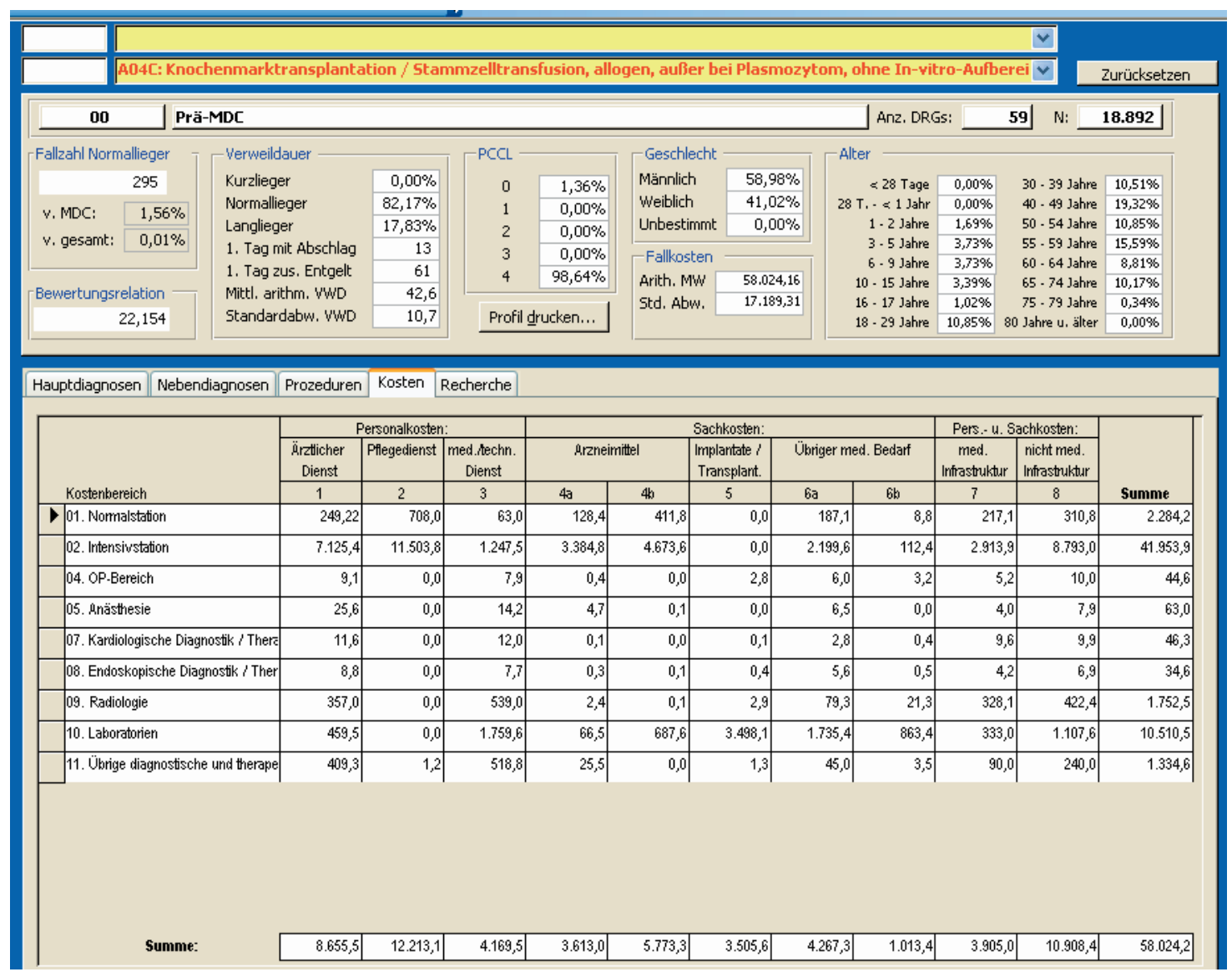

Abb. 38. Beispiel für die Kosten einer «allogenen Stammzelltransplantation» (DRG A04C).

Gesamtkosten von ca. 58000 EUR aus (Abb. 38). Für eine korrekte Abrechnung ist die Zuordnung der Intensivstation (Transplantationsmedizin? Anästhesiologie? anderer Bereich?) nötig. Auch bei einer Transplantation machen Personalkosten den Löwenanteil aus, betragen aber mit insgesamt 25000 EUR noch weniger als 50\% der Gesamtkosten. Laut Matrix entfallen bei dieser Indikation weitere 15000 EUR auf das Vorhalten der nötigen Infrastruktur. Worauf solche Kalkulationen beruhen, lässt sich im Detail schwer nachvollziehen. Die Erlösaufteilung entlang der InEK-Matrix ist deshalb unseres Erachtens nur bedingt für eine Kostenkalkulation geeignet, weil sich die Kostenaufteilung der Krankenhäuser offenbar beträchtlich unterscheidet. Sie eignet sich insbesondere nicht dafür, künftig anfallenden Personalkosten abzuschätzen, da es nicht möglich ist, die Besonderheiten des eigenen Hauses zu berücksichtigen.

Wir greifen in unserem Klinikum deshalb weiterhin auf die interne Berechnung einer gewichteten Erlösaufteilung zurück, die dann an den Vorstand und den Aufsichtsrat kommuniziert wird. Wie pflegen darüber hinaus ein Berichtswesen, das leistungsorientiert ist und Auskunft gibt etwa über Patientenzahlen, Auslastung und monatlichen CM. Dies führt nach unserer bisherigen Erfahrung weiter als die ständige Diskussion über operative Ergebnisse und die Entwicklung von Gewinnen und Verlusten.

\section{Mittelknappheit in der Onkologie - eine Umfrage unter Hämatologen und Onkologen}

\section{Referent: Stefan W. Krause, Erlangen}

Vor dem Hintergrund, dass die medizinische Versorgung in Deutschland immer teurer wird und die dafür zur Verfügung stehenden finanziellen Mittel begrenzt sind, stellt sich die Frage nach Einsparmöglichkeiten. Mit dieser Thematik befassen sich Klinikärzte bereits seit langem. Diskutiert wurden und werden verschiedene Aspekte wie die Möglichkeiten zur Einnahmesteigerung, zur weiteren Rationalisierung, aber auch zur Rationierung. Tatsache ist, dass bereits heute auf eigentlich indizierte Maßnahmen mitunter aus Kostengründen verzichtet wird, wie etwa eine Befragung von über 1000 Ärzten aus den Bereichen Kardiologie und Intensivmedizin im Jahr 2008 gezeigt hat [27]. Welche Rolle solche finanziellen Erwägungen explizit in der Onkologie spielen, ist bisher nicht näher untersucht worden. Die DGHO hat deshalb im Jahr 2011 alle per E-Mail erreichbaren Mitglieder kontaktiert mit der Bitte, einen diesbezüglichen Online-Fragebogen anonym auszufüllen. Der Fragebogen enthielt überwiegend geschlossene Fragen mit graduierten Antwortmöglichkeiten sowie Freitextfelder. Die Einladung ging an aktiv tätige Onkologen und wurde von 345 mehrheitlich fachärzt- 


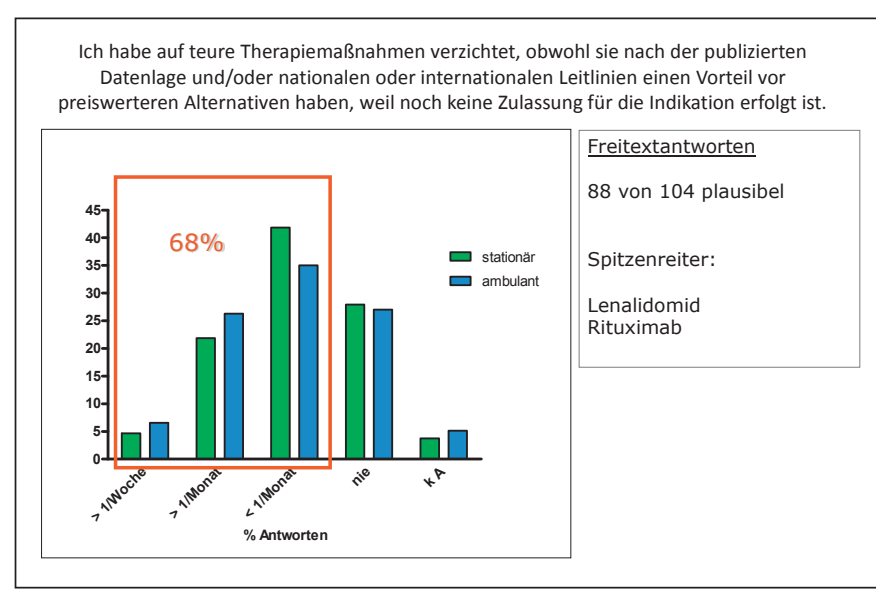

Abb. 39. Verzicht auf Therapiemaßnahmen wegen fehlender Zulassung.

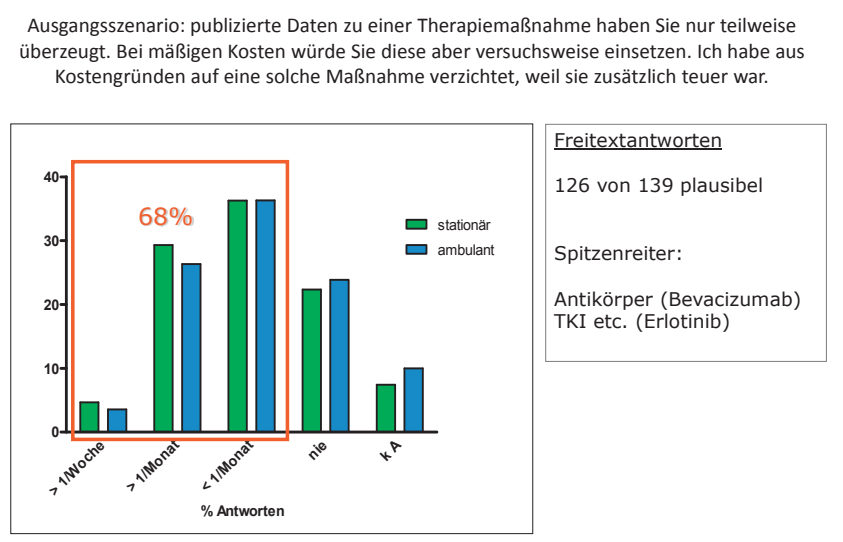

Abb. 40. Verzicht auf Therapiemaßnahmen wegen unzureichender Datenlage.

lich tätigen Kollegen ausgefüllt. Ein Teil der Ergebnisse ist inzwischen publiziert [28], eine weitere Publikation ist in Vorbereitung

\section{Entscheidung zugunsten der preiswerteren Alternative}

Im Themenbereich «Rationierung und Einsparungen» wurde unter anderem gefragt, ob und wie oft sich der Umfrageteilnehmer bei mehreren weitgehend gleichwertigen Diagnostik- oder Therapiemöglichkeiten oder Medikamenten für die/das jeweils preiswertete entschieden hat. Die meisten Kollegen tun dies häufiger als einmal pro Woche $(60 \% \mathrm{im}$ ambulanten Bereich und 70\% im stationären Bereich) sowie weitere 20 bzw. $15 \%$ mehr als einmal pro Monat. Auch bei anderen Fragen lagen die Antworten in den Bereichen stationär und ambulant ähnlich nah beieinander.

Das Statement «Ich habe eine indizierte, aber teure Maßnahme zunächst nicht angewendet und abgewartet, ob der Patient auch ohne sie auskommt» traf auf 56\% der Befragten zu (Häufigkeit: überwiegend weniger als einmal pro Monat). Diese Art der Rationierung betraf besonders Antikörper

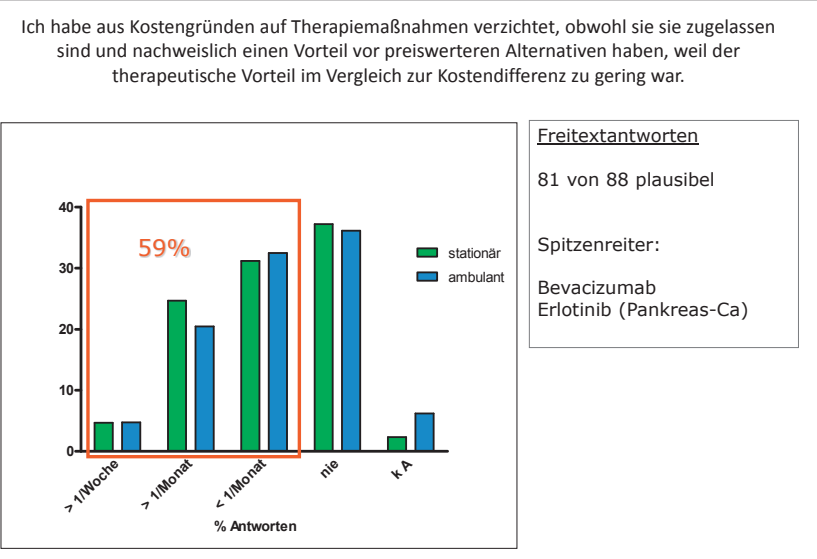

Abb. 41. Verzicht auf Therapiemaßnahmen aus Kostengründen.

\section{Prädiktoren für Rationierungsentscheidungen}

Nicht prädiktiv:

- Arme vs. reiche Bundesländer

- Private oder öffentliche Träger von Krankenhäusern

- Eigene Unzufriedenheit wg. Kostendruck

Prädiktiv:

- Eigene Einstellung zur Notwendigkeit von Rationierung

Abb. 42. Die eigene Einstellung beeinflusst das Rationierungsverhalten.

(Bevacizumab) und Tyrosinkinasehemmer, Supportiva, Antimykotika und PET-Untersuchungen.

Ein gelegentlicher Verzicht auf indizierte Maßnahmen in Zusammenhang mit Off-Label-Verordnungen (Abb. 39) und wegen unzureichender Datenlage (Abb. 40) wurde von jeweils zwei Dritteln der Befragten vorgenommen, dies galt insbesondere für den Einsatz von Lenalidomid und Rituximab im Off-Label-Bereich.

Fast $60 \%$ der Kollegen verzichteten aus Kostengründen auf Therapiemaßnahmen mit aus ihrer Sicht zu geringem therapeutischem Vorteil gegenüber preiswerteren Alternativen, allerdings taten die meisten von ihnen dies relativ selten (meist weniger als einmal pro Woche). Diese Form der Rationierung betraf unter anderem die Anwendung von Bevacizumab sowie von Erlotinib beim Pankreaskarzinom (Abb. 41).

Die Aussage «Ich habe auf eine Maßnahme verzichtet, obwohl sie nach Studienlage einen erheblichen Zusatznutzen gehabt hätte und auch zugelassen ist, weil diese Maßnahme nicht finanzierbar war» wurde letztlich aber nur von wenigen Kollegen (19\%) bestätigt. Da zudem die zugehörigen Freitextantworten teils nicht plausibel waren, scheint diese extreme Form der Rationierung tatsächlich kaum vorzukommen. 


\section{Wer soll Rationierungsentscheidungen treffen?}

2 Fragen:

- Wenn in einem Gesundheitssystem nicht alle medizinisch nützlichen Leistungen finanziert werden können, sind die für die einzelnen Patienten zuständigen Ärzte die besten Entscheider, um im Einzelfall der Behandlungssituation festzulegen, welcher Patient welche Leistungen erhält.

- Entscheidungen zum Verzicht auf medizinisch nutzbringende Leistungen sollten oberhalb der Arzt-Patienten-Ebene getroffen werden, z.B. von einer nationalen Kommission.

Abb. 43. Wer trifft die Entscheidung?

Die Überprüfung möglicher Korrelationen zwischen den getroffenen Rationierungsentscheidungen und demografischen Merkmalen ergab, dass der einzige Prädiktor für eine Entscheidung für oder gegen eine individuell vorgenommene Rationierung die jeweilige persönliche Einstellung des Befragten zum Problem der Rationierung war (Abb. 42).

Die Auswertung dieses Teils der Fragebögen hat somit ergeben, dass auch in der Onkologie - unabhängig vom Setting stationär oder ambulant - eine Rationierung am Krankenbett stattfindet, insbesondere bei Medikamenten mit einem als gering empfundenen Zusatznutzen.

\section{Wie gehen Onkologen mit der Rationierung um?}

Nach den Antworten der Befragten ist ein sparsamer Umgang mit den vorhandenen Ressourcen für die weitaus meisten der Onkologen selbstverständlich. Einhergehend damit nimmt die Auseinandersetzung mit Kostenfragen einen großen Teil der Arbeitszeit ein, befindet etwa die Hälfte der Kollegen. Ebenfalls etwa die Hälfte der Befragten gab an, gelegentlich mit den Kostenträgern über die Finanzierung einer Maßnahme zu verhandeln.

In einer weiteren Frage ging es um die Arzt-PatientenKommunikation: «Wenn eine teure Maßnahme dem Patienten bereits bekannt war und ich aus Kostengründen darauf verzichten wollte, habe ich die Kostenaspekte offen mit dem Patienten besprochen.» Dieser Aussage stimmte etwa die Hälfte der Befragten zu, während fast $30 \%$ dies nie oder fast nie tun. Wenn allerdings dem Patienten die zu erwägende Maßnahme nicht bekannt war, wurde der Verzicht auf diese Maßnahme von etwa $60 \%$ der Kollegen auch nicht thematisiert.

Der andauernde Kostendruck hat nach Angabe von etwa $70 \%$ der Onkologen die ärztliche Tätigkeit in den letzten 5 Jahren mehr oder weniger stark beeinträchtigt. Zugleich wird nach Auswegen gesucht, um trotz Mittelknappheit gute Arbeit leisten zu können. Dabei sehen rund 80\% der Befragten auch die Pharmaunternehmen in der Pflicht: Diese Kollegen meinten, es müssten mit den Herstellern innovativer Arzneimittel

\section{Individuelle Stimmen}

- eine individuelle Abwägung, welchem Pat. ich welche Behandlung 'gönne', ist völlig
falsch, subjektiv, ungerecht und hoch gefährlich
- Solange die Ärzteschaft sich regelmässig von der Industrie 'aushalten' lässt ... habe ich
Zweifel an der Objektivität
- Es ist eine Zumutung der Politik, dass kollektivethische Fragen individualethisch gelöst
werden
- Fragen müssen außerhalb des Arztzimmers und unter Beteiligung weiterer
gesellschaftlicher Gruppen (Ethik, Religion, Philosophie) bearbeitet werden!
- der Informierte bekommt das Medikament, der Uninformierte ... nicht
- Nur der behandelnde Arzt kann die Verantwortung für die Behandlung übernehmen,
eine Verschiebung zu Kommissionen oder Boards entlässt ihn nicht aus dieser
Verantwortung.
- ...glaube ich sehr wohl, dass wir als Ärzte in Kenntnis der individuellen Situation und des
Einzelschicksals am besten wissen, was noch versucht werden sollte.
- ...

Abb. 44. Persönliche Stellungsnahmen zu Rationierungsmaßnahmen.

niedrigere Preise ausgehandelt werden, falls nötig auch zwangsweise.

Die Möglichkeit, auf günstigere, wenngleich weniger effektive Therapien im Sinne einer Rationierung auszuweichen, wurde von einer knappen Mehrheit der Befragten aus der Sicht einer gesundheitspolitischen Vorgabe prinzipiell befürwortet.

Diese Positionierung zu dieser ethischen Erwägung korreliert aber nur teilweise mit den berichteten praktischen Rationierungsentscheidungen. Wie oben beschrieben, wird von jenen Befragten häufiger aus Kostengründen auf teure Maßnahmen verzichtet, die eine Rationierung auch prinzipiell befürworten. Es treffen aber auch ca. $50 \%$ jener Kollegen Entscheidungen zur Rationierung - obgleich seltener -, die ein solches Vorgehen aus grundsätzlichen Erwägungen heraus eigentlich ablehnen. Es ist zu vermuten, dass für solche Kollegen diese Entscheidungen als konfliktträchtig wahrgenommen werden.

Abschließend enthielt der Erhebungsbogen noch zwei Fragen, wer unvermeidbare Rationierungsentscheidungen treffen sollte (Abb. 43).

Von den 336 Onkologen, die sich zu diesen Fragen äußerten, war eine knappe Mehrheit für eine Verlagerung von Rationierungsentscheidungen in die Hände einer (neutralen) Kommission, der größere Teil der restlichen Kollegen votierte für eine Entscheidungsfindung auf Seiten der behandelnden Ärzte, und eine ebenfalls nicht unerhebliche Zahl von Kollegen sah sowohl neutrale Vorgaben als auch individuelle Entscheidungen für sinnvoll an. Insofern ergibt sich in der Gesamtschau ein uneinheitliches Bild, wobei von der Mehrheit der Kollegen eine zumindest teilweise Übernahme der Verantwortung durch ein neutrales Gremium bejaht wurde.

Die divergenten Auffassungen zu diesen Fragen finden sich auch in den Freitextantworten; eine Auswahl findet sich in Abbildung 44. 
Die Auswertung der Fragebögen lässt somit den Rückschluss zu, dass vielerorts eine «stille» Rationierung stattfindet, ohne die die onkologische Versorgung noch teurer wäre. Diese Rationierung wird im Rahmen einer Einzelfallentscheidung aufgrund persönlicher Einstellungen vorgenommen, da die Kriterien für den Verzicht bestimmter Maßnahmen derzeit intransparent sind. Der individuell vorgenommene Verzicht auf teure Maßnahmen aus Kostengründen wird den Patienten gegenüber nur teilweise offengelegt. Eine offene Priorisierung wird von Onkologen prinzipiell akzeptiert (allerdings mit knapper Mehrheit) wobei es unklar ist, wer Priorisierungsentscheidungen treffen soll. Es wurde außerdem deut- lich, dass die Mehrzahl der Ärzte unter dem gegenwärtigen Kostendruck leiden. Dies unterstreicht die Notwendigkeit, die Themen Priorisierung und Rationierung auch weiterhin ergebnisoffen zu debattieren.

\section{Disclosure Statement}

Bei Daniel Strech, Uwe Deh, Stephan Schmitz, Irmtraut Gürkan und Helmut Ostermann bestehen keine Interessenkonflikte.

Stefan W. Krause erhielt Forschungsunterstützung von Novartis sowie Vortragshonorare/Reiseunterstützung von Celgene, Hoffman-la-Roche, Eli Lilly, Gilead Sciences und MSD.

\section{Literatur}

1 Bundesärztekammer: Bekanntmachungen: Stellungnahme der Zentralen Kommission zur Wahrung ethischer Grundsätze in der Medizin und ihren Grenzgebieten (Zentrale Ethikkommission) bei der Bundesärztekammer zur Priorisierung medizinischer Leistungen im System der Gesetzlichen Krankenversicherung (GKV) - Zusammenfassung - (September 2007) Dtsch Arztebl 2007;104(40):A2750 / B-2430 / C-2358.

2 Sabik LM, Lie RK: Priority setting in health care: lessons from the experiences of eight countries. Int J Equity Health 2008;7:4.

3 Marckmann G: Priorisierung im Gesundheitswesen: Was können wir aus den internationalen Erfahrungen lernen? Z Evid Fortbild Qual Gesundhwes 2009;103:85-91.

4 Strech D, Marckmann G: Ethische Frameworks zur Priorisierung im Gesundheitswesen. Eine deskriptive Analyse des internationalen Status quo. Gutachten für den Verband forschender Arzneimittelhersteller e.V. (vfa), 2009.

$\checkmark 5$ Arnade J: Kostendruck und Standard. Zu den Auswirkungen finanzieller Zwänge auf den Standard sozialversicherungsrechtlicher Leistungen und den haftungsrechtlichen Behandlungsstandard, Vol. 5 . Köln, Kölner Schriften zum Medizinrecht 2010.

6 Gøtzsche PC, Olsen O: Is screening for breast cancer with mammography justifiable? Lancet 2000; 355:129-134.

7 Humphrey LL, Helfand M, Chan BK, Woolf SH: Breast cancer screening: a summary of the evidence for the U.S. Preventive Services Task Force. Ann Intern Med 2002;137:347-360.

$\checkmark$ Feinstein AR, Horwitz RI: Problems in the 'evidence' of 'evidence-based medicine'. Am J Med 1997;103:529-535.

9 Kaptchuk TJ: Effect of interpretive bias on research evidence. BMJ 2003;326:1453-1455.
Jadad AR, Cook DJ, Browman GP: A guide to interpreting discordant systematic reviews. CMAJ 1997:156:1411-1416.

11 Strech D, Tilburt J: Value judgments in the analysis and synthesis of evidence. J Clin Epidemiol 2008; 61:521-524.

12 Strech D: Werturteile in der Evidenzanalyse. Meist intransparent, oft konfliktbehaftet, nie vermeidbar. Z Evid Fortbild Qual Gesundhwes 2010;104:168176.

13 Pressemitteilung des IQWiG vom 04. Oktober 2011.

14 Sima CS, Panageas KS, Schrag D: Cancer screening among patients with advanced cancer. JAMA 2010; 304:1584-1591.

15 Kale MS, Bishop TF, Federman AD, Keyhani S: Top 5 lists top $\$ 5$ billion. Arch Intern Med 2011; 171:1856-1858.

16 Bundesärztekammer: Ulmer Papier - Gesundheitspolitische Leitsätze der Ärzteschaft. Beschluss des 111. Deutschen Ärztetages (20.-23.05.2008).

17 Brody H: From an ethics of rationing to an ethics of waste avoidance. N Engl J Med 2012;366:1949_ 1951.

18 Strech D, Danis M: How can bedside rationing be justified despite coexisting inefficiency? The need for 'benchmarks of efficiency'. J Med Ethics 2014; 40:89-93.

19 Strech D: Der Abbau von Überversorgung als Teil der ärztlichen Berufsethik. Z Gerontol Geriatr 2014;47:17-22

20 Daniels N: Just Health: Meeting Health Needs Fairly. Cambridge, Cambridge University Press, 2008.

21 Strech D, Marckmann G: Normative Versorgungsforschung. Eine orientierte Einführung (in Themen, Methoden und den Status quo in Deutschland), Gesundheits- und Sozialpolitik: Zeitschrift für das gesamte Gesundheitswesen 2012;66:8-16.
22 Gemeinsamer Bundesausschuss: Richtlinie über Maßnahmen zur Qualitätssicherung für die stationäre Versorgung von Kindern und Jugendlichen mit hämato-onkologischen Krankheiten gemäß $§ 137$ Abs. 1 Satz 1 Nr. 2 SGB V für nach § 108 SGB V zugelassene Krankenhäuser. Fassung vom: 16.05.2006 BAnz. Nr. 129 (S. 4997) vom 13.07.2006; Letzte Änderung: 06.11.2013 BAnz AT 06.12.2013 B4.

23 Gemeinsamer Bundesausschuss Richtlinie ambulante spezialfachärztliche Versorgung $§ 116 \mathrm{~b}$ SGB V - ASV-RL. Fassung vom: 21.03.2013 BAnz AT 19.07.2013 B1; Letzte Änderung: 20.02.2014 BAnz AT 25.07.2014 B1).

24 Gemeinsamer Bundesausschuss: Richtlinie ambulante spezialfachärztliche Versorgung § 116b SGB V - ASV-RL. Fassung vom: 21.03.2013 BAnz AT 19.07.2013 B1; Letzte Änderung: 20.02.2014 BAnz AT 25.07.2014 B1). In Kraft getreten am: 26.07.2014

25 Institut des Bewertungsausschusses: Bekanntmachung der Beschlüsse des ergänzten Bewertungsausschusses bzw. des ergänzten erweiterten Bewertungsausschusses nach $\S 87$ Absatz 5a SGB V. www.institut-des-bewertungsausschusses.de/ ergaenzterbewertungsausschuss/ergaenzbeschluesse. html.

26 Kassenärztliche Bundesvereinigung: Berufsmonitoring Medizinstudenten 2014.www.kbv.de/html 5724.php.

27 Strech D, Danis M, Löb M, Marckmann G: Ausmaß und Auswirkungen von Rationierung in deutschen Krankenhäusern. Ärztliche Einschätzungen aus einer repräsentativen Umfrage. Deutsche Medizinische Wochenschrift (DMW) 2009;134:1261-1266.

28 Krause SW, Schildmann J, Lotze C, Winkler EC: Rationing cancer care: a survey among the members of the german society of hematology and oncology. J Natl comp Canc Netw 2013;11(6):658-665. 